\title{
Moduli effective action in warped brane-world compactifications
}

\author{
Jaume Garriga ${ }^{1,2,3,4}$, Oriol Pujolàs ${ }^{2}$ and Takahiro Tanaka ${ }^{2,4}$
}

\author{
${ }^{1}$ Departament de Física Fonamental, Universitat de Barcelona, Diagonal 647, 08028 Barcelona, Spain \\ 2 IFAE, Departament de Física, Universitat Autònoma de Barcelona, 08193 Bellaterra (Barcelona), Spain \\ 3 Institute of Cosmology, Department of Physics and Astronomy, Tufts University, Medford MA 02155, USA \\ 4 Yukawa Institute for Theoretical Physics, Kyoto University, Kyoto 606-8502, Japan
}

(October 30, 2018)

\begin{abstract}
We consider a class of 5-D brane-world solutions with a power-law warp factor $a(y) \propto y^{q}$, and bulk dilaton with profile $\phi \propto \ln y$, where $y$ is the proper distance in the extra dimension. This class includes the Heterotic M-theory brane-world of Refs. [1,2] and the Randall-Sundrum (RS) model as a limiting case. In general, there are two moduli fields $y_{ \pm}$, corresponding to the "positions" of two branes (which live at the fixed points of an orbifold compactification). Classically, the moduli are massless, due to a scaling symmetry of the action. However, in the absence of supersymmetry, they develop an effective potential at one loop. Local terms proportional to $K_{ \pm}^{4}$, where $K_{ \pm}=q / y_{ \pm}$is the local curvature scale at the location of the corresponding brane, are needed in order to remove the divergences in the effective potential. Such terms break the scaling symmetry and hence they may act as stabilizers for the moduli. When the branes are very close to each other, the effective potential induced by massless bulk fields behaves like $V \sim d^{-4}$, where $d$ is the separation between branes. When the branes are widely separated, the potentials for each one of the moduli generically develop a "Coleman-Weinberg"-type behaviour of the form $a^{4}\left(y_{ \pm}\right) K_{ \pm}^{4} \ln \left(K_{ \pm} / \mu_{ \pm}\right)$, where $\mu_{ \pm}$are renormalization scales. In the RS case, the bulk geometry is $A d S$ and $K_{ \pm}$are equal to a constant, independent of the position of the branes, so these terms do not contribute to the mass of the moduli. However, for generic warp factor, they provide a simple stabilization mechanism. For $q \gtrsim 10$, the observed hierarchy can be naturally generated by this potential, giving the lightest modulus a mass of order $m_{-} \lesssim T e V$.
\end{abstract}

YITP-01-85

\section{INTRODUCTION}

Brane-world scenarios, where two or more parallel branes of dimension $3+1$ are embedded in a "bulk" of larger dimension have recently been used in order to construct models of considerable phenomenological interest, both in particle physics and in cosmology [3,4]. Usually, these models admit an effective 4D description at low energies, where the distances between branes are represented by scalar fields $\varphi_{i}\left(x^{\mu}\right),(\mu=0, \ldots, 3)$, called moduli.

An important example of a brane-world is the Randall-Sundrum I scenario (RS) [4], where the bulk is a slice of a 5 -dimensional anti-de Sitter space $(A d S)$ bounded by two branes of opposite tension. Matter fields may be restricted to live on the branes, but in many extensions of the original model some of them are allowed to live in the bulk too [5]. In RS there is a single modulus, called the "radion", related to the thickness of the $A d S$ slice. It is given by $\psi\left(x^{\mu}\right) \sim m_{p} \exp \left[-d\left(x^{\mu}\right) / \ell\right]$, where $d$ is the physical interbrane distance, $\ell$ is the $A d S$ curvature radius and $m_{p}$ is the Planck mass. Although in the 5D theory all fields are assumed to have physical masses comparable to $m_{p}$, the $4 \mathrm{D}$ effective mass of fields living in the negative tension brane, given by $\left.m \sim m_{p}<\psi\right\rangle$, turns out to be much smaller. This is due to a "redshift" effect induced by the AdS geometry between the branes. The exponential dependence of the $m$ on $\langle d\rangle$ can easily explain a large hierarchy between the Planck scale and the electroweak scale without the need of invoking very large numbers, which is of course one of the main assets of the RS construction.

In general, moduli fields are massless at the classical level. The reason is that they are introduced by substituting certain integration constants in the classical solutions (e.g. the interbrane distances) by slowly varying fields. Since the solutions exist for a continuous range of the integration constants, it follows that the corresponding potential for the moduli must be completely flat. Masslessness, however, is phenomenologically undesirable because it gives rise to long range scalar interactions which are severely constrained by observations, and therefore it becomes necessary to introduce a stabilization mechanism. Needless to say, this can be done in an ad hoc manner, by introducing new interactions determining the equilibrium distances between the branes. Alternatively, it is possible that a suitable mass may be generated by quantum effects.

In the absence of supersymmetry, moduli fields tend to develop an effective potential. Of course, this happens already in the simplest Kaluza-Klein (KK) compactification, and even if there are no branes [6]. A five dimensional 
field $\chi$ can be split in an infinite tower of massive KK fields, labeled by a discrete index $n$. The masses of the KK fields $m_{n}(\varphi)$ depend on the size $\varphi$ of the extra dimension, and because of that an effective potential $V(\varphi)$ is generated at one loop. From the 5D perspective, this corresponds to the (nonlocal) Casimir energy density due to the presence of compact directions.

In most cases, the self-gravity of brane and bulk matter content induce a warp in the extra dimension. In this situation the subtraction of infinities in the calculation of $V\left(\varphi_{i}\right)$ is more elaborate than it is in flat space. In particular, local terms proportional to worldvolume operators on the brane are generated, which may give rise to interesting (and sometimes surprising) physical effects. This paper follows up on our previous work [7], where we considered the particular case of the Randall-Sundrum (RS) model. A curious result [7] was the absence of logarithmic terms in the effective potential. As mentioned above, in the $4 \mathrm{D}$ effective description, the fields living in the negative tension brane have masses which are proportional to the radion expectation value. This is reminiscent of the Higgs mechanism in the standard model, and naively one might have expected the usual $4 \mathrm{D}$ logarithmic effective potential for the radion. However, we showed that a regularization which would preserve the five dimensional general covariance did not produce such logarithm. Consequently [7], it was found that the one loop effective potential is not sufficient to stabilize the radion at a phenomenologically acceptable mass in this model. Several papers have later appeared in the literature [8-11] where the effective potential is obtained in dimensional regularization, through certain subtractions corresponding to a renormalization of the brane tensions in the dimensionally extended spacetime. The results obtained in dimensional regularization were eventually found to coincide with ours, giving an indication that both procedures are in fact equivalent. The RS model, however, is somewhat special in that the bulk and branes are maximally symmetric, and all possible counterterms amount to renormalizations of the brane tensions. Therefore, in order to unveil the generic properties of the effective potential in the presence of self gravitating branes, the consideration of more general cases seems to be needed .

In this paper we shall study a more general class of warped Brane-World models, in which the bulk is no longer $A d S$. The model we consider contains a bulk "dilaton" scalar field with an exponential potential which is coupled to the 5-dimensional Einstein gravity. This provides a family of solutions with two classically massless moduli (a combination of which is the distance between branes). The solutions have a power-law warp factor $a(y) \propto y^{q}$ in place of the exponential warp of the RS model. Here $y$ is the proper distance in the extra dimension and $q$ is a constant which depends on the parameters in the Lagrangian. For $q=1 / 6$ this reduces to the Heterotic M-theory brane-world of Ref. [1], which may perhaps be relevant for the recently proposed Ekpyrotic universe scenario [2], whereas the RS model is recovered in the limit $q \rightarrow \infty$.

The plan of the paper is the following. Section II is devoted to the classical dynamics of the model. After introducing the background solution, in Section II.b we derive the action for the $4 \mathrm{D}$ moduli fields. These are massless at the classical level, due to a scaling symmetry of the 5D action which we discuss in Section II.b. The hierarchy between the Planck scale and electroweak scale is discussed in Section II.c, since in general there are some differences between the present case and the RS case. In Section III we give a brief account of quantum effects by considering the effective potential induced by a conformally invariant field. This case is rather trivial, and it properly illustrates the Casimir interaction between the branes. However, it misses the possibility of interesting local terms induced at one loop. Section IV deals with the formalism for the calculation of $V\left(\varphi_{i}\right)$ in more general cases, and contains the core of the discussion on renormalization. We start, in Section IV.A, with a digression on the specification of the measure of functional integration in a curved background. General covariance alone is not sufficient to determine the measure in the present situation, due to the presence of a nontrivial dilaton. Because of that, we shall consider a one parameter family of possibilities, related with each other through dilaton dependent conformal transformations. Sections IV.B, C and D are devoted to dimensional regularization and zeta function regularization, which are shown to give equivalent results. The explicit calculation of the effective potential in some simple cases is given in Section V. The Heterotic M-theory model is briefly discussed in Section VI. Possible consequences for the stabilization of the moduli are discussed in Section VII, and our conclusions are summarized in Section VIII. Some technical issues are left to the Appendices.

\section{THE MODEL}

The classical background we shall consider in this paper is a 5-dimensional spacetime with a nontrivial background scalar field $\phi$, which we shall refer to as the "dilaton". The fifth dimension is compactified on a $Z_{2}$ orbifold with two branes at the fixed points of the $Z_{2}$ symmetry. The action for the background fields is given by

$$
S_{b}=\frac{-1}{\kappa_{5}} \int d^{5} x \sqrt{g}\left(\mathcal{R}+\frac{1}{2}(\partial \phi)^{2}+\Lambda e^{c \phi}\right)
$$




$$
-\sigma_{+} \int d^{4} x \sqrt{g_{+}} e^{c \phi / 2}-\sigma_{-} \int d^{4} x \sqrt{g_{-}} e^{c \phi / 2},
$$

where $\mathcal{R}$ is the curvature scalar, $\kappa_{5}=16 \pi G_{5}$, where $G_{5}$ is the 5 -dimensional gravitational coupling constant. We have denoted the induced metrics on the positive and negative tension branes by $g_{\mu \nu}^{+}$and $g_{\mu \nu}^{-}$, respectively. To find a solution of the equations of motion, we make an ansatz where the 4-dimensional metric is flat,

$$
d s^{2}=d y^{2}+a^{2}(y) \eta_{\mu \nu} d x^{\mu} d x^{\nu}
$$

with a $x^{\mu}$-independent scalar field $\phi=\phi(y)$. The positive and negative branes are placed at $y=y_{+}$and $y_{-}$, respectively. Under these assumptions, the equations of motion for $(a, \phi)$ in the bulk become

$$
\begin{aligned}
& \left(\frac{\dot{a}}{a}\right)^{2}=\frac{1}{12}\left(\frac{1}{2} \dot{\phi}^{2}-U(\phi)\right), \\
& \ddot{\phi}+4 \frac{\dot{a}}{a} \dot{\phi}=U^{\prime}(\phi),
\end{aligned}
$$

where $U(\phi) \equiv \Lambda e^{c \phi}$, a dot represents differentiation with respect to $y$ and a prime represents differentiation with respect to $\phi$.

As shown in [13], there is a solution of Eqs. (3) for any value of $c$ given by

$$
\begin{aligned}
\phi & =-\sqrt{6 q} \ln \left(y / y_{0}\right), \\
a(y) & =\left(y / y_{0}\right)^{q},
\end{aligned}
$$

where

$$
q=\frac{2}{3 c^{2}}, \quad y_{0}=\sqrt{\frac{3 q(1-4 q)}{\Lambda}}
$$

(Constant rescalings of the warp factor are of course allowed, but unless otherwise stated, we shall take the convention that $a(y)=1$ at $y=y_{0}$.) Assuming $y_{-}<y_{+}$, the boundary conditions which follow from $Z_{2}$-symmetry imposed on both branes are given by

$$
\begin{aligned}
& \dot{\phi}_{ \pm}=\mp \frac{c}{4} \sigma_{ \pm} e^{(c / 2) \phi_{ \pm},} \\
& \left.6 \frac{\dot{a}}{a}\right|_{ \pm}= \pm \frac{1}{2} \kappa_{5} \sigma_{ \pm} e^{(c / 2) \phi_{ \pm}},
\end{aligned}
$$

and they are satisfied if $\sigma_{ \pm}$are tuned to

$$
\sigma_{ \pm}= \pm \frac{1}{\kappa_{5}} \sqrt{\frac{48 q \Lambda}{1-4 q}}
$$

In the absence of the branes, the spacetime (4) contains a singularity at $y=0$. Since we are considering the range between $y_{-}$and $y_{+}$, this singularity is of course inocuous. Our spacetime consists of two copies of the slice comprised between $y_{-}$and $y_{+}$, which are glued together at the branes. Hence, the 5 -th dimension is topologically an $S^{1} / Z_{2}$ orbifold.

For $q=1 / 6$ this solution is precisely the M-theory heterotic brane of Ref. [1]. On the other hand, the RS case, where the bulk is AdS and there is no scalar field, can be obtained by taking the limit $q \rightarrow \infty$ and $y_{0} \rightarrow \infty$ simultaneously, while its ratio is kept fixed,

$$
\ell=\lim _{q \rightarrow \infty} \frac{y_{0}}{q}=\sqrt{\frac{-12}{\Lambda}} .
$$

Defining $y \equiv y_{0}+y^{*}$, we find that in the limit the warp factor becomes an exponential

$$
\lim _{q \rightarrow \infty} a=e^{y^{*} / \ell},
$$

which corresponds to AdS space with curvature radius equal to $\ell$. 


\section{A. Moduli fields}

For fixed value of the coupling $c$, the solution given above contains only two physically meaningful free parameters, which are the locations of the branes $y_{-}$, and $y_{+}$. This leads to the existence of the corresponding moduli, which are massless scalar fields from the 4-dimensional point of view. In addition to these moduli, the massless sector also contains the graviton zero mode. To account for it, we generalize our metric ansatz (2) by promoting $\eta_{\mu \nu}$ to an arbitrary four-dimensional metric:

$$
d s^{2}=d y^{2}+a^{2}(y) \tilde{g}_{\mu \nu}(x) d x^{\mu} d x^{\nu}
$$

It is easy to show that $\mathcal{R}=a^{-2} \tilde{\mathcal{R}}+\mathcal{R}^{(0)}$, where $\mathcal{R}^{(0)}$ is the background Ricci scalar in five dimensions and $\tilde{\mathcal{R}}$ is the four-dimensional one. For constant values of the metric and moduli, we have a solution of the equations of motion whose action vanishes. Hence, only the terms which depend on derivatives of the metric or derivatives of the moduli will survive after the five-dimensional integration. This fact can be used in order to simplify the derivation of the effective action for the moduli, since all terms without any derivatives can be dropped.

In the bulk, all terms will cancel except for the one which is proportional to the four-dimensional Ricci scalar, $\tilde{\mathcal{R}}$. Let us consider the contribution from the branes. The metric induced on the branes is given by

$$
g_{\mu \nu}^{ \pm}=a_{ \pm}^{2}\left[\tilde{g}_{\mu \nu}+a_{ \pm}^{-2} \partial_{\mu} y_{ \pm} \partial_{\nu} y_{ \pm}\right]
$$

Here, and in what follows, the subindices \pm mean that the quantity is evaluated at the perturbed brane location. The brane tension terms in the action contain the determinant

$$
\sqrt{-g_{ \pm}}=a_{ \pm}^{4} \sqrt{-\tilde{g}}\left[1+\frac{1}{2 a_{ \pm}^{2}}\left(\tilde{\partial} y_{ \pm}\right)^{2}\right]+\cdots
$$

which induces kinetic terms for the moduli. Here, the tilde on the kinetic term indicates that the derivatives are contracted with the metric $\tilde{g}$. In fact, the five dimensional Ricci tensor $\mathcal{R}$ contains second derivatives of the metric and therefore it is singular on the brane, giving a finite contribution to the action. To handle this contribution it is simplest to introduce fiducial boundaries in the neighborhood of the branes, were we add (back to back) pairs of Gibbons-Hawking boundary terms. These have the form

$$
\frac{2}{\kappa_{5}} \int d^{4} x \sqrt{g_{ \pm}} \mathcal{K}_{ \pm}
$$

where $\mathcal{K}_{ \pm}$is the trace of the extrinsic curvature of the fiducial boundary near each one of the branes. The action is separated into two parts. The first one is a "bulk" part, consisting of an integral over two copies of an open set which excludes the branes, $\left(y_{-}+\epsilon\right)<y<\left(y_{+}-\epsilon\right)$, plus terms of the form (11) at the boundaries $y=y_{-}+\epsilon$ and $y=y_{+}-\epsilon$. Then there is a "brane" part, which includes an integral of the action over the infinitessimal open sets of thickness $\epsilon$ around the branes, suplemented with terms of the form (11) at the boundaries of these open sets (these "brane" boundary terms sit back to back with the ones used in order to bound the "bulk", and have opposite sign relative to them, since the normal to the fiducial boundary has opposite sign on each side of the boundary. Thus the total effect of the boundary terms is to add zero to the action). As is well known, through integration by parts the boundary terms absorb the second derivatives in the Einstein term $\mathcal{R}$. Hence, the singular contribution from the gravity kinetic term on the brane disappears, and in the limit $\epsilon \rightarrow 0$, the only contribution to the action from the interval of width $2 \epsilon$ around the branes (together with the added boundary terms), is from the brane tension term itself, but not from the gravity kinetic term. On the other hand, the bulk contribution has to be supplemented with the boundary terms (11).

The boundary terms can be evaluated as follows. Consider, for instance the hypersurface which is located at $y=y_{+}\left(x^{\sigma}\right)$. In terms of the new coordinate $\hat{y}=y-y_{+}\left(x^{\sigma}\right)$, the brane is at $\hat{y}=0$, and the metric can be written as

$$
d s^{2}=N^{2} d \hat{y}^{2}+g_{\mu \nu}^{+}\left(N^{\mu} d \hat{y}+d x^{\mu}\right)\left(N^{\nu} d \hat{y}+d x^{\nu}\right)
$$

where we have introduced the lapse function $N^{2}=1-g_{\mu \nu}^{+} N^{\mu} N^{\nu}$, and the shift vector $N^{\mu}=g^{+\mu \nu} y_{, \nu}$. Then, the trace of the extrinsic curvature is given by

$$
\begin{aligned}
\int d x^{4} \sqrt{-g_{+}} \mathcal{K}_{+} & =\int d x^{4} \frac{\sqrt{-g_{+}}}{2 N}\left[g^{+\mu \nu} \partial_{\hat{y}} g_{\mu \nu}^{+}-2 N^{\mu} \mid \mu\right] \\
& =\int d x^{4} \frac{\sqrt{g_{+}}}{2 N}\left[\frac{\dot{a}}{a} g^{+\mu \nu} \tilde{g}_{\mu \nu}-\frac{N^{\mu} \partial_{\mu} N^{2}}{N^{2}}\right]
\end{aligned}
$$




$$
\approx 4 \int\left(\frac{\dot{a}}{a}\right)_{+} a_{+}^{4} \sqrt{-\tilde{g}}\left[1+\frac{3}{4 a_{+}^{2}}\left(\tilde{\partial} y_{+}\right)^{2}\right],
$$

where the vertical line means a covariant differentiation with respect to the induced metric $g_{\mu \nu}^{+}$. We neglected the terms which are higher order in in derivatives of the modulus $y_{+}$. As mentioned before, the subindices \pm mean that the quantities are evaluated at the actual position of the brane, and the expression is in fact nonperturbative in the positions $y_{ \pm}$themselves (although not in the derivatives).

Substituting the previous expressions into the action (1), with the addition of the extrinsic curvature terms, and using the background equations of motion we find

$$
\begin{aligned}
S_{b}=\int d^{4} x \sqrt{-\tilde{g}}[ & -\left(2 \int_{y_{-}}^{y_{+}} d y a^{2}\right) \frac{1}{\kappa_{5}} \tilde{\mathcal{R}} \\
& \left.+\frac{1}{2} \sigma_{+} e^{(c / 2) \phi_{+}} a_{+}^{2}\left(\tilde{\partial} y_{+}\right)^{2}+\frac{1}{2} \sigma_{-} e^{(c / 2) \phi_{-}} a_{-}^{2}\left(\tilde{\partial} y_{-}\right)^{2}\right] .
\end{aligned}
$$

This can be rewritten as

$$
S_{b}=\frac{-1}{16 \pi G} \int d^{4} x \sqrt{-\tilde{g}}\left\{\left(\varphi_{+}^{2}-\varphi_{-}^{2}\right) \tilde{\mathcal{R}}-\frac{6 q}{q+1 / 2}\left[\left(\tilde{\partial} \varphi_{+}\right)^{2}-\left(\tilde{\partial} \varphi_{-}\right)^{2}\right]\right\} .
$$

Here we have introduced

$$
\varphi_{ \pm} \equiv\left(\frac{y_{ \pm}}{y_{0}}\right)^{q+1 / 2}
$$

and the four dimensional Newton's constant $G$ given by

$$
G=\left(q+\frac{1}{2}\right) \frac{G_{5}}{y_{0}} .
$$

The modulus corresponding to the positive tension brane has a kinetic term with the "wrong" sign. However, this does not necessarily signal an instability, because it is written in a Brans-Dicke frame. One may go to the Einstein frame by a conformal transformation. It is convenient to introduce the new moduli $\varphi$ and $\psi$ through [2]

$$
\varphi_{+}=\varphi \cosh \psi, \quad \varphi_{-}=\varphi \sinh \psi,
$$

and to define the new metric

$$
\hat{g}_{\mu \nu}=\varphi^{2} \tilde{g}_{\mu \nu}
$$

It is then straightforward to show that $\varphi^{2} \sqrt{\tilde{g}} \tilde{\mathcal{R}}=\sqrt{-\hat{g}}\left[\hat{R}+6 \varphi^{-2}(\hat{\partial} \varphi)^{2}\right]$. Substituting into the background action (15), we have

$$
S_{b}=\frac{-1}{16 \pi G} \int d^{4} x \sqrt{-\hat{g}}\left\{\hat{\mathcal{R}}+\frac{6}{1+2 q} \frac{(\hat{\partial} \varphi)^{2}}{\varphi^{2}}+\frac{12 q}{1+2 q}(\hat{\partial} \psi)^{2}\right\} .
$$

Therefore, both moduli have positive kinetic terms in the Einstein frame. At the classical level, the moduli are massless, but as we shall see in the following Sections, a potential term of the form

$$
\delta S=-\int d^{4} x V(\varphi, \psi) \equiv-\int d^{4} x \sqrt{-\hat{g}} \hat{V}(\varphi, \psi),
$$

is generated at one loop, which should be added to (17).

In the RS limit $q \rightarrow \infty$ [see Eq. (9)] the kinetic term for one of the moduli disappears. This is to be expected, because the bulk is the maximally symmetric AdS space. In this case only the relative position of the branes $y_{+}-y_{-}$ is physically meaningful and the other modulus can be gauged away (see also [12] for a recent discussion of this case).

Matter does not couple universally to the 4D Einstein metric $\hat{g}_{\mu \nu}$. Rather, it will couple to combinations of the metric and the moduli fields. For completeness, in Appendix A we give an alternative form for the moduli action in terms of the induced metrics on either brane, to which brane matter fields are universally coupled. 


\section{B. Scaling symmetry}

Since we are interested in the effective potential for the moduli, it is perhaps pertinent to start by asking why these fields are massless at the classical level. The reason is that under the global transformation

$$
\begin{gathered}
g_{a b} \rightarrow T^{2} g_{a b}, \\
\phi \rightarrow \phi-(2 / c) \ln T,
\end{gathered}
$$

the action (1) scales by a constant factor

$$
S_{b} \rightarrow T^{3} S_{b}
$$

Here $g_{a b}$ is the metric appearing in the action (1). Acting on a solution with one brane, the transformation simply moves the brane to a different location. Hence, all brane locations are allowed, from which the masslessness of the moduli follows. However, we should hasten to add that this is just a global scaling symmetry which need not survive quantum corrections.

It is interesting to observe that by means of a conformal transformation, we may construct a new metric $g_{a b}^{(s)}$ which is invariant under the scaling symmetry

$$
g_{a b}^{(s)}=e^{c \phi} g_{a b} .
$$

In terms of this new metric the action takes the form

$$
\begin{aligned}
S_{b} & =\frac{-1}{\kappa_{5}} \int d^{5} x \sqrt{g^{(s)}} e^{-3 c \phi / 2}\left(\mathcal{R}^{(s)}+\left[(1 / 2)-3 c^{2}\right]\left(\partial^{(s)} \phi\right)^{2}+\Lambda\right) \\
& -\sigma_{+} \int d^{4} x \sqrt{g_{+}^{(s)}} e^{-3 c \phi / 2}-\sigma_{-} \int d^{4} x \sqrt{g_{-}^{(s)}} e^{-3 c \phi / 2} .
\end{aligned}
$$

Now, the symmetry is a mere shift in $\phi$. Moreover, with our background solutions for $g_{a b}$ and $\phi$, the metric $g_{a b}^{(s)}$ is just AdS, as can be easily shown from (21) and (4).

For certain discrete values of $c$, the action (22) can be obtained from dimensional reduction of $(5+n)$ dimensional pure gravity with a cosmological term $\Lambda$, where the additional $n$ dimensions are toroidal [24]. In this case, the factor $e^{-3 c \phi / 2}$ is the overall scale of the internal $n$-dimensional volume, and the value of $c$ is given by $c^{2}=2 / 3 q$, with

$$
q=\frac{n+3}{n} .
$$

It is not surprising, then, that the metric $g_{a b}^{(s)}$ in $(22)$ corresponds to the "external" components of a $5+n$ dimensional anti de Sitter space, since the starting point is in fact pure gravity in $(5+n)$ dimensions with a negative cosmological term. The calculation of quantum corrections in this higher dimensional space, and its relation with the calculation of quantum corrections in the effective 5D theory which we consider in this paper, will be reported in a separate publication [24].

\section{The hierarchy}

As mentioned in the introduction, one of the motivations for studying brane-world scenarios has been the search for a geometric origin of the hierarchy between the effective Planck scale $m_{p}$ and the electroweak scale. In the 5D description, all matter fields are assumed to have masses which are close to the cut-off scale of the theory $M \equiv G_{5}^{-1 / 3}$. And yet, with the help of an exponential warp factor (as in the RS model) it is easy to generate a hierarchy of the order of $m_{p} / m \sim 10^{16}$. Here $m$ is the effective mass of fields which live on the negative tension brane, as "seen" in the effective four dimensional description [4]. In this subsection we shall review this mechanism, including the case of a warp factor with arbitrary power $q$, since there are some minor differences with the RS case.

The effective four-dimensional Planck mass is given by

$$
m_{p}^{2}=\frac{2}{1+2 q} M^{3} y_{+}\left[1-\left(\frac{y_{-}}{y_{+}}\right)^{2 q+1}\right]
$$


where $M$ is the 5-dimensional Planck mass, as can be seen from Eqs. (15) and (16)], where, without loss of generality, we have taken $y_{+}=y_{0}$. Here, and for the rest of this Section, we shall follow standard practice and refer all physical quantities to the measurements of clocks and rods located on the positive tension brane.

Let us now consider the mass scales of fields which live on the branes. We expect these fields to couple not only to the metric, but also to the background dilaton $\phi$. There are many possible forms for this coupling, but it seems reasonable to restrict attention to those which respect the scaling symmetry (19-20). For a free scalar field $\Psi$ which lives on the negative tension brane, and whose mass parameter is comparable to the cut-off scale, the action takes the form

$$
S_{\Psi}=-\frac{1}{2} \int \sqrt{g_{-}^{(s)}} F^{2}(\phi)\left[g_{-}^{(s) \mu \nu} \partial_{\mu} \Psi \partial_{\nu} \Psi+\alpha M^{2} \Psi^{2}\right] .
$$

Here we have introduced a fudge factor $\alpha$ to allow for an intrinsic mass which is slightly lower than the cut-off scale. The function $F(\phi)$ can be reabsorbed in a redefinition of $\Psi$, and thus the relevant warp which determines the hierarchy between mass scales on the positive and in the negative tension branes is the one corresponding to the metric $g^{(s)}$. Our field will be perceived from the point of view of the 4D effective theory as having a mass squared of order

$$
m^{2} \sim \alpha M^{2}\left(\frac{y_{-}}{y_{+}}\right)^{2 q-2}
$$

Notice that there are two different factors which determine the hierarchy between $m$ and $m_{p}$. The first one is the warp factor $\left(y_{-} / y_{+}\right)^{q-1}$ appearing in Eq. (25), which "redshifts" the mass scales of particles on the negative tension brane (except for $q<1$, in which case the particles on the negative tension brane appear to be heavier than those on the positive tension brane). This generates the hierarchy in the RS model. The second one is the possibly large volume of the internal space, which enhances the effective Planck scale with respect to the cut-off scale [see Eq. (23)]. This generates the hierarchy in the ADD model [3] with large extra dimensions. Considering both effects, the hierarchy $h$ is given by

$$
h^{2}=\frac{m^{2}}{m_{p}^{2}} \sim \alpha \frac{1+2 q}{2} \frac{1}{M y_{+}}\left(\frac{y_{-}}{y_{+}}\right)^{2 q-2} .
$$

It is known that without a warp factor, it is not possible to generate the desired hierarchy from a single extra dimension, since its size would have to be astronomical. An interesting question is what is the minimum value of the exponent $q$ which would be sufficient in order to generate a ratio $m / m_{p} \sim 10^{-16}$. The best we can do is to take the curvature scale $\left(y_{+} / q\right)$ slightly below the millimeter scale,

$$
\left(y_{+} / q\right) \lesssim m_{p}(\mathrm{TeV})^{-2} \sim m m .
$$

in order to pass the short distance tests on deviations from Newton's law. On the other hand, we also need

$$
\left(y_{-} / q\right) \gtrsim M^{-1},
$$

since for smaller values of $y_{-}$the curvature becomes comparable to the cutoff scale $M$ and the theory cannot be trusted ${ }^{1}$. Substituting in (23) we have $M^{3} \gtrsim m_{p}(\mathrm{TeV})^{2}$ and $\left(y_{-} / y_{+}\right) \gtrsim\left(m_{p} / \mathrm{TeV}\right)^{-4 / 3}$, which leads to

$$
10^{-32} \sim \frac{m^{2}}{m_{p}^{2}} \gtrsim \alpha\left(\frac{T e V}{m_{p}}\right)^{\frac{4(2 q-1)}{3}} .
$$

Hence, a warp factor with exponent $q \geq 5 / 4$ may account for the observed hierarchy with a single extra dimension, but it appears that this cannot be done for lower values of $q .{ }^{2}$ In particular, the Heterotic M-theory model, with $q=1 / 6$, does not seem to allow for such possibility.

\footnotetext{
${ }^{1}$ One should also bear in mind that the scale $M$ might itself be a "derived" quantity, as it happens for instance with the Planck mass in theories with additional large extra dimensions. In this case, the true cut-off scale may well be below $M$. Hence, the lower bound (27) on $y$ - should just be considered a necessary condition for the low energy description not to break down. ${ }^{2}$ Except, of course, by giving up the assumption that the Lagrangian of matter on the branes should scale in the same way as the rest of the classical action [see the discussion around Eq. (24)]. If we allow any coupling of $\phi$ to the mass term for $\Phi$, then any hierarchy can be easily generated for any value of $q$.
} 


\section{EFFECTIVE POTENTIAL INDUCED BY BULK CONFORMAL FIELDS}

Before embarking on a detailed discussion of renormalization, we shall consider in this Section the case of a conformal bulk field $\chi$. This case is rather easy to handle, and it is useful in illustrating the non-local contribution of the vacuum energy to the masses of the moduli. Unfortunately, it misses possibly interesting effects which may arise due to renormalization of local operators on the brane, whose discussion we defer to the next sections.

Following the discussion given in $\S$ III of [7], we define the conformal coordinates by

$$
z \equiv\left|\int \frac{d y}{a(y)}\right|=\frac{y_{0}}{|1-q|}\left(\frac{y}{y_{0}}\right)^{1-q}
$$

and we rewrite the metric as

$$
d s^{2}=a^{2}(z)\left(d z^{2}+\eta_{\mu \nu} d x^{\mu} d x^{\nu}\right), \quad a(z)=\left(z / z_{0}\right)^{\beta},
$$

where

$$
\beta=\frac{q}{1-q}, \quad z_{0}=\frac{y_{0}}{|1-q|} .
$$

Here we should mention that the direction of increasing $z$ does not coincide with the direction of increasing $y$ when $q>1$.

The Casimir energy density $\rho$ of the conformally coupled scalar field in this spacetime is related to its counterpart in a flat spacetime with a compactified extra dimension ( $a=1$ in the above metric) by a conformal transformation. The relation is $\rho=a^{-5} \rho_{0}$, where $\rho_{0}$ is the flat space value:

$$
\rho_{0}=\frac{V_{0}}{2\left|z_{+}-z_{-}\right|}=\mp \frac{A}{2\left|z_{+}-z_{-}\right|^{5}} ; \quad A \equiv \frac{\pi^{2}}{32} \zeta_{R}^{\prime}(-4) \approx 2.46 \cdot 10^{-3} \text {. }
$$

Here $z_{+}$and $z_{-}$are the position of the positive and the negative tension branes in the conformal coordinate $z$. The double signs in (32) refer to bosons or fermions respectively. Then, we find that the contribution of a conformally coupled scalar field to the effective potential per unit co-moving volume is given by

$$
V\left(z_{+}, z_{-}\right)=\sqrt{-\hat{g}} \hat{V}=2 \int a^{5}(z) \rho d z=\mp \frac{A}{\left|z_{+}-z_{-}\right|^{4}} .
$$

Here, $\hat{V}$ is the effective potential per unit "physical" volume (i.e. the volume as measured with the Einstein metric $\hat{g})$, to be inserted in (17). In terms of the moduli fields $\varphi$ and $\psi$ we have

$$
\hat{V}(\varphi, \psi)=\mp B \Lambda^{2} \varphi^{-12 \gamma}\left[(\cosh \psi)^{3 \gamma-1}-(\sinh \psi)^{3 \gamma-1}\right]^{-4},
$$

where we have defined

$$
B=\frac{A(1-q)^{4}}{9 q^{2}(1-4 q)^{2}}, \quad \gamma=\frac{1}{1+2 q}
$$

and we have used $\sqrt{\hat{g}}=\varphi^{4}$ for the background solution. It should be mentioned that local terms induced by quantum corrections may be added to (34), both due to fields which live on the branes as well as from non-conformal bulk fields. A discussion of these terms is deferred to the next section.

In the $\mathrm{RS}$ case $\gamma \rightarrow 0$ the $\varphi$ dependence in (34) disappears, and we recover the results of [7]. We have

$$
\hat{V}_{R S}= \pm \frac{A}{16 \ell^{4}} e^{4 \psi} \sinh ^{4} 2 \psi
$$

For $\psi \ll 1$, the field $\psi$ corresponds to the hierarchy between scales on both branes:

$$
\tanh \psi \approx \psi=\frac{a_{-}}{a_{+}} \sim \frac{T e V}{m_{P l}} \ll 1,
$$

where $m_{P l} \sim 10^{19} \mathrm{GeV}$ is the Planck mass. In the second equation we assume that the warp is responsible for the hierarchy between Planck and electroweak scales. In this case we have

$$
\hat{V}_{R S}(\psi) \approx \frac{A}{\ell^{4}} \psi^{4}[1+4 \psi+\ldots] \sim A(T e V)^{4} .
$$


Here we have assumed that the AdS radius is not far below the Planck scale, in which case the contribution to the effective potential is of electroweak order. This huge contribution must somehow be cancelled by some other term in order to have an acceptable four dimensional cosmological constant. Also, the slope of the potential has to be cancelled at the place where the radion sits. These two conditions can be imposed if we allow for a finite renormalization of the brane tensions, which contribute proportionally to $a_{+}^{4} \propto \cosh ^{4} \psi \approx 1$ and $a_{-}^{4} \propto \sinh ^{4} \psi \approx \psi^{4}$ for the positive and negative tension branes respectively. With these additions we have

$$
\hat{V}_{R S}(\psi)=A \ell^{-4}\left[c_{1}+c_{2} \psi^{4}+4 \psi^{5}+\ldots\right],
$$

where $c_{1}$ and $c_{2}$ are undetermined constants which can only be fixed by experiment. The "renormalization" condition $\partial_{\psi} V(\psi)=0$ at the observed value of the radion $\psi=\psi_{\text {obs }} \sim T e V / m_{P l}$ forces $c_{2} \sim \psi_{\text {obs }}$. Hence the mass of the radion induced by this quantum correction is given by [7]

$$
m_{\psi}^{2} \approx A \frac{\psi_{o b s}^{3}}{\ell^{4} m_{P l}^{2}} \sim A \psi_{o b s}(T e V)^{2} .
$$

Again, in the last equality, we have taken $\ell$ to be similar to the Planck scale. The radion mass is much lower than the electroweak scale and hence it is not enough for a phenomenologically viable stabilization of the radion. The reason for such as small mass is that $V(\psi)$ is polynomial for small $\psi$, with the first power being $\psi^{4}$. The situation would be different if the potential took the form $\psi^{4} \log (\psi)$ as in the Coleman-Weinberg case. Then, the induced mass $m_{\psi}^{2}$ would be of the same order as the induced potential. Remarkably, the absence of logarithmic terms seems to extend to the contribution from massive fields too [8], and so in the RS case it appears that the radion cannot acquire a phenomenologically interesting mass solely from one loop quantum effects.

For the more general case of a power law warp factor, the possibility of an efficient stabilization by quantum effects will depend on the nature of the local operators which are induced by quantum corrections. We now turn to a discussion of this subject.

\section{EFFECTIVE POTENTIAL IN THE GENERAL CASE}

In this Section we set up the framework for computing the contribution to the 1-loop effective potential from a scalar field $\chi$ propagating in the bulk with a generic mass term, which may include couplings to the curvature of spacetime as well as couplings to the background dilaton $\phi$. The effective potential for the moduli $y_{ \pm}$will be defined as usual in terms of a Gaussian path integral around the background solution. Before presenting the actual calculation, however, a digression on the choice of the measure of integration will be useful.

\section{A. Specification of the functional measure}

A quantum field theory is defined not just by the classical Lagrangian, but it is also necessary to specify the measure of functional integration. The latter is usually prescribed by demanding certain symmetries or invariances. For instance, for scalar fields in curved space, invariance under diffeomorphisms is an obvious requirement. If gravity were the only background field, then this requirement would suffice to uniquely define Gaussian integration around that background. On the other hand, if there are fields other than gravity with a nontrivial profile (such as our dilaton $\phi)$, then there is a wide class of possible choices, related to each other by dilaton dependent conformal transformations. All choices within this class are equally good from the point of view of diffeomorphism invariance. Strictly speaking, however, they are inequivalent due to the well known conformal anomaly.

To be definite, let us concentrate in the simple case of a bulk scalar field $\chi$ with canonical kinetic term. The (Euclidean) action for this field is given by

$$
S[\chi]=\frac{1}{2} \int d^{D} x \sqrt{g} \chi P \chi,
$$

where we have introduced the covariant operator

$$
P=-\left(\square_{g}+E\right) .
$$

Here $\square_{g}$ is the d'Alembertian operator associated with the metric $g_{a b}$, and $E=E\left[g_{a b}, \phi\right]$ is a generic "mass" term. Typically, this takes the form $E=-m^{2}-\xi \mathcal{R}_{g}$, where $m$ is a constant mass, $\mathcal{R}_{g}$ is the curvature scalar and $\xi$ is an arbitrary coupling. Throughout this Section we shall leave $E$ unspecified. 
A volume measure $\mathcal{D} \chi$ in field space $\mathcal{F}$ can be found from a metric $G_{x y}$ on $\mathcal{F}$, through the relation:

$$
\mathcal{D} \chi=\sqrt{G} \prod_{x} d \chi^{x}
$$

Here, the spacetime coordinates $x$ and $y$ are considered as continuous labels for the coordinates $\chi^{x} \equiv \chi(x)$ of the infinite dimensional space $\mathcal{F}$, and $G$ is the determinant of $G_{x y}$. To specify $G_{x y}$, we note that a natural definition of a scalar product in the space of field variations $\delta \chi$ can be given in terms of the spacetime measure $d \mu(x)$, through the relation

$$
<\delta \chi_{1}, \delta \chi_{2}>_{\mu} \equiv \iint d \mu(x) d \mu(y) G_{x y} \delta \chi_{1}^{x} \delta \chi_{2}^{y} \equiv \int d \mu(x) \delta \chi_{1}(x) \delta \chi_{2}(x) .
$$

We denote field variations by $\delta \chi$ just to emphasize that we are refering to elements of the tangent space. More precisely, $\delta \chi=\delta \chi^{x} e_{x}$, where $e_{x}=\partial / \partial \chi^{x}$ is the coordinate basis of the tangent space at the point $p$ which corresponds to the background solution. In a Riemannian spacetime, the invariant measure is given by

$$
d \mu(x)=\sqrt{g(x)} d^{D} x,
$$

where $g$ is the determinant of $g_{a b}$, and $D$ is the dimension. The implicit definition of $G_{x y}$ given above is just the identity $\delta_{\mu}(x, y)$ with respect to $d \mu$ integration,

$$
G_{x y}=\delta_{\mu}(x, y)=\frac{\delta^{(n)}(x-y)}{\sqrt{g(x)}} .
$$

It is convenient to express the field variations in an orthonormal basis $\chi_{n}$, with $\left\langle\chi_{n}, \chi_{m}\right\rangle=\delta_{n m}$, so that $\delta \chi(x)=$ $\sum_{n} c^{n} \chi_{n}(x)$. In this basis, the components of the field variation are $c^{n}$, and the metric is just the usual delta function (the continuous or the discrete delta function depending on whether the normalization of $\chi_{n}$ is continuous or discrete):

$$
G_{n m}=\delta_{n m}
$$

Substituting in (37), we have

$$
\mathcal{D} \chi=\prod_{n} d c^{n}
$$

It should be clear from the previous discussion that the definition of $\mathcal{D} \chi$ is associated with a natural definition of $d \mu(x)$. However, in the problem under consideration in this paper, the choice of $d \mu$ is not unique. In our case, there is a nontrivial dilaton field $\phi$, and we can consider a whole class of spacetime measures of the form

$$
d \mu_{\theta}(x)=\sqrt{g_{\theta}} d^{D} x=\Omega_{\theta}^{D}(\phi) \sqrt{g} d^{D} x,
$$

which correspond to conformally related metrics

$$
g_{a b}^{\theta}=\Omega_{\theta}^{2} g_{a b},
$$

for an arbitrary function $\Omega_{\theta}(\phi)$ (we shall use some specific choices of this function in the next subsection). In the presence of a dilaton, the coupling to gravity is not universal and it is not clear which one of these metrics should be considered more physical. To proceed, it is convenient to define the operator $P_{\theta}$ associated with the metric $g_{a b}^{\theta}$ by

$$
\Omega_{\theta}^{(D-2) / 2} P_{\theta} \Omega_{\theta}^{(2-D) / 2}=\Omega_{\theta}^{-2} P,
$$

where $P$ was introduced after Eq. (36). This operator can be written in covariant form as

$$
P_{\theta}=-\left(\square_{\theta}+E_{\theta}\right),
$$

where

$$
E_{\theta}=\left(\frac{D-2}{2}\right) \square_{\theta} \ln \Omega_{\theta}-\left(\frac{D-2}{2}\right)^{2} g_{\theta}^{a b} \partial_{a} \ln \Omega_{\theta} \partial_{b} \ln \Omega_{\theta}+\Omega_{\theta}^{-2} E,
$$


and $\square_{\theta}$ is the covariant d'Alembertian corresponding to $g_{a b}^{\theta}$. Introducing $\chi_{\theta} \equiv \Omega_{\theta}^{(2-D) / 2} \chi$, the action for the scalar field can be expressed as

$$
S[\chi]=\frac{1}{2} \int d^{D} x \sqrt{g_{\theta}} \chi_{\theta} P_{\theta} \chi_{\theta} .
$$

In terms of $g_{a b}^{\theta}$ the field $\chi_{\theta}$ has a perfectly canonical and covariant kinetic term.

Thus, the same arguments which lead to (39) can now be used in order to find the natural line element in field space associated with the spacetime measure $d \mu_{\theta}(x)$ :

$$
d \mathcal{S}_{\theta}^{2}=\iint d \mu_{\theta}(x) d \mu_{\theta}(y) G_{x y}^{\theta} d \chi_{\theta}^{x} d \chi_{\theta}^{y}+\ldots=\int d^{D} x \sqrt{g_{\theta}(x)}\left(d \chi_{\theta}(x)\right)^{2}+\ldots
$$

Here, the ellipsis denote the omitted terms which correspond to variations of other fields in the theory (in particular, these include the variations of the gravitational field and the dilaton). Let us compare this line element with the one considered above

$$
d \mathcal{S}^{2}=\iint d \mu(x) d \mu(y) G_{x y} d \chi^{x} d \chi^{y}+\ldots=\int d^{D} x \sqrt{g(x)}(d \chi(x))^{2}+\ldots
$$

For field variations where $\chi$ changes but the rest of the fields (metric, dilaton, etc.) are constant, we have

$$
d \mathcal{S}_{\theta}^{2}=\int d^{D} x \Omega_{\theta}^{2} \sqrt{g(x)}(d \chi(x))^{2} \neq d \mathcal{S}^{2} \quad\left(g, \phi, \ldots=\text { const.; } d \chi^{x} \neq 0\right),
$$

and therefore $d \mathcal{S}_{\theta}^{2} \neq d \mathcal{S}^{2}$ in general. Of course, the corresponding measures of integration will also be different. In the basis $\left\{\chi_{\theta n}\right\}$ which is orthonormal with respect $d \mu_{\theta}$, the field variation can be expanded as $\delta \chi_{\theta}(x)=\sum_{n} c_{\theta}^{n} \chi_{\theta n}$, and the new measure takes the form

$$
(\mathcal{D} \chi)_{\theta}=\prod_{n} d c_{\theta}^{n}
$$

Using $\chi_{\theta m}=\Omega_{\theta}^{-D / 2} \chi_{m}$, it is straigtforward to show that $c^{m}=M_{n}^{m} c_{\theta}^{n}$, where $M_{n}^{m}=<\chi_{m}, \Omega_{\theta}^{-1} \chi_{n}>_{\mu} \equiv\left(\Omega_{\theta}^{-1}\right)_{n}^{m}$. Hence the two measures (40) and (44) are related by

$$
\mathcal{D} \chi=J_{\theta}(\mathcal{D} \chi)_{\theta},
$$

where the Jacobian is formally given by

$$
J_{\theta}=\operatorname{det}\left(\Omega_{\theta}^{-1}\right)=\exp \left[-\operatorname{Tr} \ln \Omega_{\theta}\right]
$$

In the last step we have used the formal definition of the $L_{2}$ trace: ${ }^{3}$ :

$$
\operatorname{Tr}[\mathcal{O}]=\sum_{m} \int d^{D} x g^{1 / 2} \chi_{m}\left(\mathcal{O} \chi_{m}\right)=\sum_{m} \int d^{D} x g_{\theta}{ }^{1 / 2} \chi_{\theta m}\left(\mathcal{O} \chi_{\theta m}\right) .
$$

The trace is well defined if the diagonal matrix elements of the operator $\mathcal{O}$ decay sufficiently fast at large momenta. Unfortunately, the diagonal matrix elements of $\ln \Omega_{\theta}$ do not decay at all at large $m$, and so the trace is ill defined unless we introduce a regulator. We will address this question below, where we will explicitly define what we mean by $J_{\theta}$.

Perhaps we should add, for the sake of clarity, that the difference between the line elements $d \mathcal{S}^{2}$ and $d \mathcal{S}_{\theta}^{2}$, and consequently the difference between the associated measures, is not due to field redefinitions. Both objects are different, but since they are defined geometrically, they are both invariant under field redefinitions (in the same sense that any line element is invariant under coordinate transformations). Rather, the relation (46) expresses the

\footnotetext{
${ }^{3}$ The definition of the trace is robust, in the sense that it is independent on the metric one uses in order to define the orthonormal basis, as long as the corresponding measures are in the same $L_{2}$ class. This will be the case, for instance, if the metrics are related by a conformal factor which is bounded above and below on the manifold.
} 
well known conformal anomaly. The measure is not invariant under conformal transformations because these do not correspond to a change of coordinates in field space $\mathcal{F}$. They correspond to a change of the spacetime metric and consequently to a change of the metric on $\mathcal{F}$. This sort of ambiguity does not arise when we consider scalar fields in flat space. Consider an action with a general kinetic term of the form:

$$
S=\int d^{D} x G_{A B}\left(\phi^{C}\right) \eta^{a b} \partial_{a} \phi^{A} \partial_{b} \phi^{B}+\ldots
$$

A natural line element in field space can be obtained by "stripping off" the flat metric $\eta^{a b}$ and replacing the partial derivatives with differentials of the fields:

$$
d \mathcal{S}^{2}=\int d^{D} x G_{A B}\left(\phi^{C}\right) d \phi^{A} d \phi^{B} .
$$

This procedure cannot be transported into a curved space, because in erasing the factor $g^{a b}$ from the kinetic term, it makes a difference what exactly we have chosen to call the metric of spacetime: $g_{a b}$ or $g_{a b}^{\theta}$ (this is, by the way, the reason why the factor of $\Omega_{\theta}^{2}$ appears in (43)). Usually, flat space definitions can be generalized to curved space through the principle of general covariance: objects should be defined geometrically, and they should reduce to their flat space definition when the spacetime metric is flat. The question is, however, which object should be considered to play the role of spacetime metric, so that we know when to call it flat. Physically, too, one should expect that a preferred spacetime metric should play a role in regularizing and renormalizing the theory. Suppose that we attempt to regularize with a physical cut-off, so that all degrees of freedom beyond a certain scale are ignored. In our background (which is conformally flat) a constant physical cut-off scale corresponds to a different co-moving scales at different places. The relation between physical and co-moving scale is of course given by the metric, and therefore it can make a difference which one we use.

Since we have a classical scaling symmetry in the gravity and dilaton sector, one could argue that $g_{a b}^{(s)}$, which is invariant under scaling (see Section II.b), is the preferred physical metric. 4 However, even in this case the divergent part of the effective potential will not respect the scaling symmetry, and consequently we need to introduce counterterms with the "wrong" scaling behaviour. Hence, in what follows, we shall take the conservative attitude that the measure is determined in the context of a more fundamental theory (from which our 5-D effective action is derived), and we shall formally consider on equal footing all choices associated with metrics in the conformal class of $g_{a b}$, including of course $g_{a b}^{(s)}$. As we shall see, the difference between these choices amounts to the addition of local terms in the effective potential.

The contribution of the field $\chi$ to the renormalized effective potential $V_{\theta}$ per unit co-moving volume parallel to the branes is given by:

$$
\exp \left[-\mathcal{A}\left(V_{\theta}+V_{\theta}^{d i v}\right)\right] \equiv \int(\mathcal{D} \chi)_{\theta} e^{-S[\chi]}=\left(\operatorname{det} P_{\theta}\right)^{-1 / 2}
$$

where $\mathcal{A}$ is the co-moving volume under consideration and we have used (42) and the measure (44) to express the gaussian integral as a determinant. The term $V_{\theta}^{\text {div }}$ is a local counterterm which, in dimensional regularization, needs to be subtracted from the regularized effective potential (its explicit form will be given in the coming sections). In zeta function regularization, the left hand side is already finite, and $V_{\theta}^{\text {div }}$ is unnecessary (it corresponds to a finite renormalization of couplings). Eq. (47) can be written as

$$
V_{\theta} \equiv \frac{1}{2 \mathcal{A}} \ln \left(\operatorname{det} P_{\theta}\right)-V_{\theta}^{d i v} .
$$

Eq. (45) suggests the notation

$$
V_{\theta}=\frac{1}{2 \mathcal{A}} \ln (\operatorname{det} P)-V_{\theta}^{d i v}+\frac{1}{\mathcal{A}} \ln J_{\theta} .
$$

The reader should be aware, however, that the definition of the Jacobian in Eq. (46) is only formal because the trace in the r.h.s. of this equation is ill defined. For that reason, it is not clear that Eq. (49) would hold with the definition

\footnotetext{
${ }^{4}$ Note, in particular, that the overall scaling factor of the action (1) under (19) and (20) depends on the spacetime dimension, and hence the symmetry itself is different when we change the dimension. By contrast, the scaling of (22) remains the same in any dimension.
} 
(46), after substituting determinants by traces and applying any kind of regularization to the formally divergent traces. To avoid misinterpretations, in the discussions that follow we shall take $J_{\theta}$ to be defined by Eq. (49), that is

$$
\ln J_{\theta} \equiv \frac{1}{2}\left[\ln \left(\operatorname{det} P_{\theta}\right)-\ln (\operatorname{det} P)\right],
$$

where the expression in the right hand side is to be calculated in some regularization scheme.

The way the $\theta$ dependence of $V_{\theta}$ arises is very different in different regularization schemes. In Eq. (49), the determinant of $P$ is independent of $\theta$ (we recall that this operator corresponds to the choice $\Omega_{\theta}=1$ ). In dimensional regularization, $\ln J_{\theta}$ vanishes, but the divergent term $V_{\theta}^{\text {div }}$ which is subtracted from $\ln (\operatorname{det} P$ ) depends on the choice of physical metric $g_{a b}^{\theta}$. On the other hand, in zeta function regularization, $\ln (\operatorname{det} P)$ is finite and $V^{d i v}$ does not play a role (in any case, any finite renormalization does not introduce a dependence in $\theta$ ). Rather, in this case, the dependence on $\theta$ comes from $\ln J_{\theta}$, which does not vanish in this regularization scheme. In both cases, the $\theta$ dependence of $V_{\theta}$ is the same.

As we shall see, this dependence can be cast in the form of local operators on the branes, and therefore the ambiguity in the choice of the integration measure can also be understood as modification of the classical action. It should be noted, however, that the local operators which result from a shift in $\theta$ have different form than the terms arising from the usual shift in the renormalization constant $\mu$ which inevitably crops up in the regularized traces. In the cases we shall consider, the latter will take the form $K^{4}\left(y_{ \pm}\right)$, where $K$ denotes terms which behave like the extrinsic curvature of the branes at the positions $y_{ \pm}$. On the other hand, the $\theta$-dependent terms behave as $K^{4}\left(y_{ \pm}\right) \phi\left(y_{ \pm}\right)$. Since $K(y)$ behaves like the inverse of $y$ whereas $\phi(y)$ behaves logarithmically with $y$, these terms will give rise to Coleman-Weinberg type potentials for the moduli.

\section{B. Conformal transformations and the KK spectrum}

The direct evaluation of the determinant of $P_{\theta}$ appearing in Eq. (47) turns out to be rather impractical, due to the complicated form of the implicit equation which defines its eigenvalues. For actual calculations it is convenient to work with a conformally related operator $P_{0}$ whose eigenvalues will be related to the KK masses.

Following [7], we introduce a one-parameter family of metrics which interpolate between a fictitious flat spacetime and any of the metrics in the conformal class of the Einstein metric:

$$
g_{a b}^{\theta}=\Omega_{\theta}^{2}(\phi) g_{a b}
$$

where $\theta$ parametrizes a path in the space of conformal factors. For definiteness we shall restrict attention to conformal factors $\Omega_{\theta}(\phi)$ which have an exponential dependence on the dilaton:

$$
\Omega_{\theta}(z)=e^{(1-\theta) \phi / 3 c}=\left(\frac{z}{z_{0}}\right)^{\beta(\theta-1)} .
$$

With this choice, $\theta=0$ represents flat space and $\theta=1$ corresponds to the Einstein frame metric (30). For $\theta=-1 / \beta$,

the metric $g_{a b}^{\theta}$ coincides with the metric $g_{a b}^{(s)}$ introduced in Subsection II.B, which is invariant under the scaling transformation (as mentioned before, this metric corresponds to a five dimensional AdS space, with curvature radius given by $\left.z_{0}\right)$.

The operator $P_{0} \equiv P_{\theta=0}$ is the wave operator for the KK modes which one would use in a four-dimensional description. The Lorentzian equation of motion $P \chi=0$ can be written as

$$
P_{0} \chi_{0}=0,
$$

where

$$
P_{0}=-\square_{D-1}+\hat{M}^{2}(z) .
$$

Here $\square_{D-1}$ is the flat space d'Alembertian along the branes, and

$$
\hat{M}^{2} \equiv-\partial_{z}^{2}-E_{0},
$$

is the Schrödinger operator whose eigenvalues are commonly referred to as the KK masses $m_{n}$ :

$$
\hat{M}^{2}(z) \chi_{0, n}(z)=m_{n}^{2} \chi_{0, n}(z) .
$$


The interesting feature of (53) is that it separates into a four-dimensional part and a $z$ dependent part. A mode of the form $\chi_{0}=e^{i k_{\mu} x^{\mu}} \chi_{0, n}$ will solve the equation of motion (53) provided that the dispersion relation

$$
k_{\mu} k^{\mu}+m_{n}^{2}=0
$$

is satisfied, and hence modes labeled by $n$ behave as four-dimensional massive particles. Technically, the advantage of working with $P_{0}$ is that its (Euclidean) eigenvalues $\lambda_{n, k}=k_{\mu} k^{\mu}+m_{n}^{2}$ separate as a sum of a four-dimensional part plus the eigenvalue of the Schrödinger problem in the fifth direction.

In the following subsections, we shall discuss how $\operatorname{det} P_{0}$ is related to the determinant of our interest, $\operatorname{det} P$, or more generally to $\operatorname{det} P_{\theta}$. For completeness, and in order to illustrate practical methods for calculating the effective potential, we shall consider dimensional regularization and zeta function regularization. Both methods will lead to identical results.

\section{Dimensional regularization}

A naive reduction to flat four-dimensional space suggests that the effective potential can be obtained as a sum over the KK tower:

$$
V^{D} \equiv \mu^{\epsilon} \sum_{n} \frac{1}{2} \int \frac{d^{D-1} k}{(2 \pi)^{D-1}} \log \left(\frac{k^{2}+m_{n}^{2}\left(\varphi_{i}, D\right)}{\mu^{2}}\right) .
$$

Here $D=4+1-\epsilon$ is the dimension of spacetime, and we have added $(-\epsilon)$ dimensions parallel to the brane. The renormalized effective potential should then be given by an expression of the form

$$
V\left(\varphi_{i}\right)=V^{D}-V^{d i v}
$$

and the question is what to use for the divergent subtraction $V^{\text {div }}$. Since Eq. (55) is similar to an ordinary effective potential in 4-dimensional flat space ${ }^{5}$, one might imagine that $V$ can be obtained from $V^{D}$ just by dropping the pole term, proportional to $1 / \epsilon$; but this is not true for warped compactifications

$$
V\left(\varphi_{i}\right) \neq V^{D}-(\text { pole term) }
$$

The point is that the theory is five dimensional and the spacetime is curved, and this fact must be taken into account in the process of renormalization.

Rather than proceeding heuristically from (56), we must take the definition of the effective potential Eq. (48) as our starting point, where it is understood that the formally divergent trace must be regularized and renormalized. In order to identify the divergent quantity to be subtracted, we shall use standard heat kernel expansion techniques. Let us introduce the dimensionally regularized expressions [14]

$$
V_{\theta}^{D} \equiv \frac{\mu^{\epsilon}}{2 \mathcal{A}} \operatorname{Tr} \ln \left(\frac{P_{\theta}(D)}{\mu^{2}}\right)=-\frac{\mu^{\epsilon}}{2 \mathcal{A}} \lim _{s \rightarrow 0} \partial_{s} \zeta_{\theta}(s, D),
$$

where

$$
\zeta_{\theta}(s, D)=\operatorname{Tr}\left[\left(\frac{P_{\theta}(D)}{\mu^{2}}\right)^{-s}\right]=\frac{2 \mu^{2 s}}{\Gamma(s)} \int_{0}^{\infty} \frac{d \xi}{\xi} \xi^{2 s} \operatorname{Tr}\left[e^{-\xi^{2} P_{\theta}(D)}\right]
$$

It should be noted that the operator $P_{\theta}$ is positive and therefore the integral is well behaved at large $\xi$.

As is well known, the regularized potential $V_{\theta}^{D}$ contains a pole divergence in the limit $D \rightarrow 5$. To see that this is the case, one introduces the asymptotic expansion of the trace for small $\xi[15,19,20]$,

$$
\operatorname{Tr}\left[f e^{-\xi^{2} P_{\theta}(D)}\right] \sim \sum_{n=0}^{\infty} \xi^{n-D} a_{n / 2}^{D}\left(f, P_{\theta}\right)
$$

\footnotetext{
${ }^{5}$ It should be mentioned also that each KK contribution in Eq.(55) is not just like a flat space contribution, because in warped compactifications the KK masses $m_{n}(\varphi, D)$ depend on the number of external dimensions parallel to the brane.
} 
where $a_{n / 2}^{D}$ are the so-called generalized Seeley-De Witt coefficients. In (59) we have introduced the arbitrary smearing function $f(x)$. This is unnecessary for the present discussion, but it will be useful later on. For $n \leq 5$ their explicit form is known for a wide class of covariant operators, which includes our $P_{\theta}$. They are finite and can be constructed from local invariants (terms constructed from the metric, the mass term $E_{\theta}$ and the smearing function $f$ ), integrated over spacetime. For even $n$, they receive contributions from the bulk and from the branes, whereas for odd $n$ they are made out of invariants on the boundary branes only.

For definiteness, let us focus on the simplest case of a Dirichlet scalar field, satisfying ${ }^{6}$

$$
\chi\left(z_{ \pm}\right)=0 .
$$

We can use the result found in [16-18] to compute the Seeley-De Witt coefficients for a Dirichlet field with a bulk operator $P=-(\square+E)$. The lowest order ones for odd $n$ are given by:

$$
\begin{gathered}
a_{1 / 2}^{D}(f, P)=\frac{-(4 \pi)^{\frac{(1-D)}{2}}}{4} \sum_{i= \pm} \int_{y_{i}} \sqrt{g_{i}} f(x) d^{D-1} x \\
a_{3 / 2}^{D}(f, P)=\frac{-(4 \pi)^{\frac{(1-D)}{2}}}{384} \sum_{i= \pm} \int_{y_{i}} \sqrt{g_{i}} d^{D-1} x\left\{f\left(96 E-16 \mathcal{R}+8 \mathcal{R}_{y y}+7 \mathcal{K}^{2}-10 \mathcal{K}_{\mu \nu} \mathcal{K}^{\mu \nu}\right)+O\left(f_{; y}, f_{; y y}\right)\right\} .
\end{gathered}
$$

The most relevant for our purposes will be $a_{5 / 2}^{D}$ :

$$
\begin{aligned}
& a_{5 / 2}^{D}(f, P)=\frac{-(4 \pi)^{\frac{(1-D)}{2}}}{5760} \sum_{i= \pm} \int_{y_{i}} \sqrt{g_{i}} d^{D-1} x\left\{f \left(720 E^{2}-450 \mathcal{K} E_{; y}+360 E_{; y y}\right.\right. \\
& +15\left(7 \mathcal{K}^{2}-10 \mathcal{K}_{\mu \nu} \mathcal{K}^{\mu \nu}+8 \mathcal{R}_{y y}-16 \mathcal{R}\right) E \\
& +20 \mathcal{R}^{2}-48 \square \mathcal{R}-17 \mathcal{R}_{y y}^{2}-8 \mathcal{R}_{a b} \mathcal{R}^{a b}+8 \mathcal{R}_{a b c d} \mathcal{R}^{a b c d} \\
& -20 \mathcal{R}_{y y} \mathcal{R}+16 \mathcal{R}_{y y} \mathcal{R}-10 \mathcal{R}_{y y} \mathcal{R}_{y y}-12 \mathcal{R}_{; y y}-15 \mathcal{R}_{y y ; y y} \\
& -16 \mathcal{K}_{\mu \nu} \mathcal{K}^{\nu \rho} \mathcal{R}_{\rho}^{\mu}-32 \mathcal{K}^{\mu \nu} \mathcal{K}^{\rho \sigma} \mathcal{R}_{\mu \rho \nu \sigma}+\frac{215}{8} \mathcal{K}_{\mu \nu} \mathcal{K}^{\mu \nu} \mathcal{R}_{y y} \\
& +25 \mathcal{K}_{\mu \nu} \mathcal{K}^{\mu \nu} \mathcal{R}+\frac{47}{2} \mathcal{K}_{\mu \nu} \mathcal{K}_{\rho}^{\nu} \mathcal{R}^{\rho}{ }_{y \mu y}+\frac{215}{16} \mathcal{R}_{y y} \mathcal{K}^{2} \\
& -\frac{35}{2} \mathcal{R} \mathcal{K}^{2}-14 \mathcal{K}_{\mu \nu} \mathcal{R}^{\mu \nu} \mathcal{K}-\frac{49}{4} \mathcal{K}^{\mu \nu} \mathcal{R}_{\mu y \nu y} \mathcal{K}+42 \mathcal{R}_{; y} \mathcal{K} \\
& -\frac{65}{128} \mathcal{K}^{4}-\frac{141}{32} \mathcal{K}_{\mu \nu} \mathcal{K}^{\mu \nu} \mathcal{K}^{2}+\frac{17}{2} \mathcal{K}_{\mu \nu} \mathcal{K}^{\nu \rho} \mathcal{K}_{\rho}^{\mu} \mathcal{K} \\
& \left.\left.+\frac{777}{32}\left(\mathcal{K}^{\mu \nu} \mathcal{K}_{\mu \nu}\right)^{2}-\frac{327}{8} \mathcal{K}_{\mu \nu} \mathcal{K}^{\nu \rho} \mathcal{K}_{\rho \sigma} \mathcal{K}^{\mu \sigma}\right)+O\left(f_{; y}, \ldots, f_{; \text {yyy }}\right)\right\} .
\end{aligned}
$$

Our notation is as follows. $E$ is a general scalar function, $\mathcal{R}_{b c d}^{a}=+\Gamma_{b c, d}^{a}-\cdots$ is the Riemann tensor, $\mathcal{R}_{b c}=\mathcal{R}_{b a c}^{a}$ is the Ricci tensor and $\mathcal{R}=\mathcal{R}_{a b} g^{a b}$ is the curvature scalar. The extrinsic curvature is given by $\mathcal{K}_{\mu \nu} \equiv(1 / 2) \partial_{y} g_{\mu \nu}$, where $g_{\mu \nu}(y)$ is the induced metric on $y$-constant hypersurfaces, and $\mathcal{K}=\mathcal{K}_{\mu \nu} g^{\mu \nu}$. The vector normal to the boundary is $\partial_{y}$ so the normal components are simply the $y$ components. The $a, b, \cdots$ indices run over the extra coordinate, and over the directions tangential to the branes, $\mu, \nu, \cdots$. The omitted terms, represented by $O\left(f_{; y}, \ldots\right)$, are linear combinations of the derivatives of $f$ with coefficients which depend on $\mathcal{K}_{\mu \nu}, E$ and its derivatives.

As mentioned above, the integral (58) is well behaved for large $\xi$. For small $\xi$, the integral is convergent for $2 s>D$, as can be seen from the asymptotic expansion (59). In the end, we have to consider the limit $s \rightarrow 0$, and so we must keep track of divergences which may arise in this limit. For this purpose, it is convenient to separate the integral into a small $\xi$ region, with $\xi<\Lambda$, and a large $\xi$ region with $\xi>\Lambda$, where $\Lambda$ is some arbitrary cut-off. Substituting (59) into (58), we can explicitly perform the integration in the small $\xi$ region for $2 s>D$. This gives

\footnotetext{
${ }^{6}$ In $§$ III we considered an even parity scalar field, which obeys Neumann boundary conditions. In fact, in the conformally trivial case we would get the same result for an odd parity field since the spectrum in the flat space problem is the same. The only difference is the presence of a zero mode in the even parity case, which does not contribute to the result (34).
} 


$$
\zeta(s, D) \sim 2 \frac{\mu^{2 s}}{\Gamma(s)}\left\{\sum_{n=0}^{\infty} \frac{\Lambda^{n-D+2 s}}{n-D+2 s} a_{n / 2}^{D}\left(P_{\theta}\right)+\int_{\Lambda}^{\infty} \frac{d \xi}{\xi} \xi^{2 s} \operatorname{Tr}\left[e^{-\xi^{2} P_{\theta}(D)}\right]\right\},
$$

where we have used the standard notation

$$
a_{n / 2}^{D}\left(P_{\theta}\right)=a_{n / 2}^{D}\left(f=1, P_{\theta}\right) .
$$

The second term in curly brackets is perfectly finite for all values of $s$. Analytically continuing and taking the derivative with respect to $s$ at $s=0$, we have

$$
\zeta^{\prime}(0, D) \sim \sum_{n=0}^{\infty} \frac{2 \Lambda^{n-D}}{n-D} a_{n / 2}^{D}\left(P_{\theta}\right)+\text { finite }
$$

where the last term is just twice the integral in (64) evaluated at $s=0$. Introducing the regulator $\epsilon=5-D$, the ultraviolet divergent part of $V_{\theta}^{D}$ is thus given by

$$
V_{\theta}^{d i v}=-\frac{1}{\epsilon \mathcal{A}} a_{5 / 2}^{D}\left(P_{\theta}\right)
$$

The divergence is removed by renormalizing the couplings in front of the invariants which make up the coefficient $a_{5 / 2}^{D}$, and so this infinite term can be dropped. The renormalized effective potential of our interest is therefore given by

$$
V_{\theta}=\lim _{D \rightarrow 5}\left[V_{\theta}^{D}-V_{\theta}^{d i v}\right]
$$

To proceed, we need to calculate $V_{\theta}^{D}$, which in principle requires calculating a trace which involves the eigenvalues of $P_{\theta}$, and as mentioned above, these are not related in any simple way to the KK masses.

However, it turns out that the dimensionally regularized $V_{\theta}^{D}$ is independent of $\theta$ when $D$ is not an integer. The dependence of $V_{\theta}^{D}$ on $\theta$ can be found in the following way. First we note that

$$
\partial_{\theta} \operatorname{Tr}\left[e^{-\xi^{2} P_{\theta}}\right]=\operatorname{Tr}\left[2 \xi^{2} f_{\theta}(x) \Omega_{\theta}^{-2} P e^{-\xi^{2} P_{\theta}}\right]=-\xi \partial_{\xi} \operatorname{Tr}\left[f_{\theta}(x) e^{-\xi^{2} P_{\theta}}\right]
$$

where we have introduced

$$
f_{\theta} \equiv \partial_{\theta} \ln \Omega_{\theta},
$$

and the cyclic property of the trace was used. The above relation enables us to find the dependence of $V_{\theta}^{D}$ on the conformal factor:

$$
\partial_{\theta} \lim _{s \rightarrow 0} \partial_{s} \zeta_{\theta}(s, D)=\lim _{s \rightarrow 0} \partial_{s} \frac{2 \mu^{2 s}}{\Gamma(s)} \int_{0}^{\infty} d \xi \xi^{2 s} \partial_{\xi} \operatorname{Tr}\left[-f_{\theta} e^{-\xi^{2} P_{\theta}}\right] .
$$

As with the expansion (64) we may again introduce the regulator $\Lambda$ and separate the integral into a large $\xi$ part with $\xi>\Lambda$, which is finite and a small $\xi$ part with $\xi<\Lambda$ which contains the divergent ultraviolet behaviour. Assuming that $2 s>D$ and integrating by parts, the resulting integrals in the small $\xi$ region can be performed explicitly and we have

$$
\partial_{\theta} \lim _{s \rightarrow 0} \partial_{s} \zeta_{\theta}(s, D) \sim \lim _{s \rightarrow 0} \partial_{s} \frac{4 s \mu^{2 s}}{\Gamma(s)}\left[\sum_{n=0}^{\infty} \frac{\Lambda^{n-D+2 s}}{n-D+2 s} a_{n / 2}^{D}\left(f_{\theta}, P_{\theta}\right)+\text { finite }\right]
$$

As before, the last term just indicates the integral in the large $\xi$ region. Provided that $D$ is not an integer, all terms in square brackets remain finite at small $s$, and so the right hand side of (70) vanishes. Hence, we find that

$$
\partial_{\theta} V_{\theta}^{D}=0, \quad(D \neq \text { integer }) .
$$

In other words, the dimensionally regularized determinant of $P_{\theta}$ coincides with the dimensionally regularized determinant of $P_{0}$, and we have

$$
V_{\theta}^{D}=V_{0}^{D} \equiv V^{D} \equiv \sum_{n} \mu^{\epsilon} \frac{1}{2} \int \frac{d^{D-1} k}{(2 \pi)^{D-1}} \log \left(\frac{k^{2}+m_{n}^{2}\left(\varphi_{i}, D\right)}{\mu^{2}}\right), \quad(D \neq \text { integer }) .
$$


As was anticipated, we find that $\ln J_{\theta}$ vanishes in the dimensional regularization presented here in the sense given in (50).

Finally, from (67) and (72), the renormalized effective potential is given by

$$
V_{\theta}(\varphi)=\lim _{D \rightarrow 5}\left[V^{D}-\frac{1}{(D-5)} \frac{1}{\mathcal{A}} a_{5 / 2}^{D}\left(P_{\theta}\right)\right],
$$

where the Seeley-De Witt coefficient $a_{5 / 2}^{D}$ is given in (63) with $f=1$. The above equation bears the ambiguity in the choice of integration measure in the second term in square brackets. Different values of $\theta$ give different results. If we take $g_{a b}$ as the preferred metric, then we should use $\theta=1$, whereas if we take $g_{a b}^{(s)}$ as the preferred metric, we should use $\theta=-1 / \beta$. As we shall see in the next subsection, when we set $D=5$ the coefficient $a_{5 / 2}\left(P_{\theta}\right)$ is also independent of $\theta$. Hence, the pole term in the second term in (73) is independent of $\theta$, as it should, in order to cancel the pole in $V^{D}$. However, the finite part does depend on the choice of $\theta$.

The right hand side of (73) is ready for explicit evaluation, which is deferred to the next section. We shall now turn our attention to the equivalent method of zeta function regularization.

\section{Zeta function regularization}

The method of zeta function regularization exploits the fact that the formal expression for the effective potential (57) is finite if the limit $D \rightarrow 5$ is taken before the limit $s \rightarrow 0$. This can be seen from Eq. (64), where the term with $n=5$ is finite if we set $D=5$ before taking the derivative with respect to $s$ and setting $s \rightarrow 0$. Clearly, the change in the order of the limits simply removes the divergent term $V^{\text {div }}$ given in (66) and it reproduces the results obtained by the method of dimensional regularization (up to finite renormalization terms which are proportional to the geometric invariant $\left.a_{5 / 2}^{D=5}\left(P_{\theta}\right)\right)$.

In zeta function regularization we define

$$
V_{\theta} \equiv-\frac{1}{2 \mathcal{A}} \ln \left(\operatorname{det} P_{\theta}\right) \equiv-\frac{1}{2 \mathcal{A}} \lim _{s \rightarrow 0} \partial_{s} \zeta_{\theta}(s),
$$

where $\zeta_{\theta}(s) \equiv \zeta_{\theta}(s, D=5)$ [see Eq. (58)]. As in the case of dimensional regularization, it is more convenient to calculate $V_{0}$ than $V_{\theta}$ since the eigenvalues of $P_{0}$ are related to the spectrum of KK masses. An important difference with dimensional regularization is that

$$
-2 \mathcal{A} \partial_{\theta} V_{\theta}=\partial_{\theta} \zeta_{\theta}^{\prime}(0)=2 a_{5 / 2}\left(f_{\theta}, P_{\theta}\right) \neq 0,
$$

a result which we already encountered in Ref. [7] (see also [16]). This can be seen from (70). If we set $D=5$ from the very beginning, the term with $n=5$ in Eq. (70) is linear in $s$, and its derivative with respect to $s$ does not vanish in the limit $s \rightarrow 0$. Here, and in what follows, we use the notation

$$
a_{n / 2} \equiv \lim _{D \rightarrow 5} a_{n / 2}^{D} .
$$

Integrating along the conformal path parameterized by $\theta$, we can relate the effective potential per unit comoving volume $V_{\theta}$, with the "flat space" effective potential $V_{0}$ as

$$
V_{\theta}=V_{0}-\frac{1}{\mathcal{A}} \int_{0}^{\theta} d \theta^{\prime} a_{5 / 2}\left(f_{\theta^{\prime}}, P_{\theta^{\prime}}\right)
$$

The general expression for $a_{5 / 2}\left(f_{\theta}, P_{\theta}\right)$ which applies to our case has been derived by Kirsten [16]. In Ref. [7] we evaluated the integral in (75) for the Randall-Sundrum case, in order to obtain $V_{1}$ from $V_{0}$. Here we shall present an alternative expression for this integral which does not require the knowledge of $a_{5 / 2}\left(f_{\theta}, P_{\theta}\right)$, but only the knowledge of $a_{5 / 2}^{D}\left(P_{\theta}\right)$ for dimension $D=5-\epsilon$. This will also illustrate the relation between the method of zeta function regularization and the method of dimensional regularization.

From the asymptotic expansion of the first and the last terms in Eq. (68), we have [21]

$$
\partial_{\theta} a_{n / 2}^{D}\left(P_{\theta}\right)=(D-n) a_{n / 2}^{D}\left(f_{\theta}, P_{\theta}\right) .
$$

Integrating over $\theta$, we get 


$$
(D-5) \int_{0}^{\theta} a_{5 / 2}^{D}\left(f_{\theta^{\prime}}, P_{\theta^{\prime}}\right) d \theta^{\prime}=a_{5 / 2}^{D}\left(P_{\theta}\right)-a_{5 / 2}^{D}\left(P_{0}\right) .
$$

Writing $D=5-\epsilon$, we have

$$
V_{\theta}-V_{0}=-\frac{1}{\mathcal{A}} \int_{0}^{\theta} a_{5 / 2}\left(f_{\theta^{\prime}}, P_{\theta^{\prime}}\right) d \theta^{\prime}=\frac{1}{\epsilon \mathcal{A}}\left[a_{5 / 2}^{D}\left(P_{\theta}\right)-a_{5 / 2}^{D}\left(P_{0}\right)\right] .
$$

Note that, from (66) and (73), the previous equation can also be written as

$$
V^{D} \equiv V_{\theta}+V_{\theta}^{d i v}=V_{0}+V_{0}^{d i v}
$$

This equation simply expresses the fact that the dimensionally regularized $V^{D}$ is independent of the conformal parameter $\theta$, as we had shown in the previous Subsection [see e.g. Eq. (71)].

From $(76)$, with $D=n=5$, one finds that the coefficient $a_{5 / 2}\left(P_{\theta}\right)$ is conformally invariant [21], and therefore

$$
a_{5 / 2}\left(P_{\theta}\right)=a_{5 / 2}\left(P_{0}\right)
$$

Substituting this into (78), we obtain

$$
\int_{0}^{\theta} a_{5 / 2}\left(f_{\theta^{\prime}}, P_{\theta^{\prime}}\right) d \theta^{\prime}=\left.\frac{d}{d D} a_{5 / 2}^{D}\left(P_{\theta}\right)\right|_{D=5}-\left.\frac{d}{d D} a_{5 / 2}^{D}\left(P_{0}\right)\right|_{D=5} .
$$

Thus, the integral in (75) can be evaluated in two different ways. One is by using the explicit expression of $a_{5 / 2}(f, P)$ given by Kirsten [16]. The other is by taking the derivative of the coefficients $a_{5 / 2}^{D}\left(P_{\theta}\right)$, given in $(63)$ with $f=1$, with respect to the dimension. [Note that the terms which are linear in derivatives of $f$, which we have just indicated symbolically in (63), disappear when $f$ is a constant].

The quantity discussed above is nothing but the $\theta$ dependence induced by the choice of integration measure. In the sense of (50), we have

$$
\ln J_{\theta}=\frac{1}{3 c \mathcal{A}} \int_{\theta}^{1} d \theta^{\prime} a_{5 / 2}\left(\phi, P_{\theta^{\prime}}\right)
$$

where we have used $f_{\theta}=\partial_{\theta} \ln \Omega_{\theta}=(1-\theta) \phi / 3 c$. Clearly, the effect of this factor is just adding local terms expressed solely in terms of $\phi$ and the metric to the classical action. The dependence of these terms is different from the change which results from a rescaling of the renormalization parameter $\mu$. This corresponds to a shift in the coefficient of local terms proportional to $a_{5 / 2}(P)$.

\section{EXPLICIT EVALUATION}

For simplicity we shall restrict attention to the case of massless fields with arbitrary coupling to the curvature:

$$
E=-\xi \mathcal{R}_{g}
$$

and with Dirichlet boundary conditions. Here we shall use the method of dimensional regularization. Zeta function regularization is discussed in the Appendix.

The eigenmodes in (54) are given by

$$
\hat{\chi}_{n} \equiv z^{1 / 2}\left(A_{1} J_{\nu}\left(m_{n} z\right)+A_{2} Y_{\nu}\left(m_{n} z\right)\right) .
$$

The index of the Bessel functions is given by

$$
\nu(D)=\frac{1}{2} \sqrt{1-4(D-1) \beta[(D-2) \beta-2]\left(\xi-\xi_{c}(D)\right)},
$$

where

$$
\xi_{c}(D)=\frac{1}{4} \frac{D-2}{D-1}
$$


is the conformal coupling in dimension $D$. Imposing the boundary conditions (60) on both branes, we obtain the equation that defines implicitly the discrete spectrum of $m_{n}$,

$$
F\left(\tilde{m}_{n}\right)=J_{\nu}\left(\tilde{m}_{n} \eta\right) Y_{\nu}\left(\tilde{m}_{n}\right)-Y_{\nu}\left(\tilde{m}_{n} \eta\right) J_{\nu}\left(\tilde{m}_{n}\right)=0
$$

where we have defined

$$
\tilde{m}_{n}=m_{n} z_{-}, \quad \eta=\frac{z_{+}}{z_{-}}
$$

The zeros of $F$ are all real if $\nu$ is real and $\eta$ is positive. Since $\eta$ is positive, the reality condition of $\nu$ guarantees that all the KK masses are real. This provides a constraint for the possible values of $\xi$ depending on $q$,

$$
\begin{array}{ll}
\xi \geq \frac{-(1-4 q)^{2}}{16 q(2-5 q)}, & q \leq 2 / 5, \\
\xi \leq \frac{-(1-4 q)^{2}}{16 q(2-5 q)}, & q \geq 2 / 5,
\end{array}
$$

where we have used $D=5$. Note that the values of $\xi$ comprised between the minimal and the conformal coupling are allowed for any value of $q$.

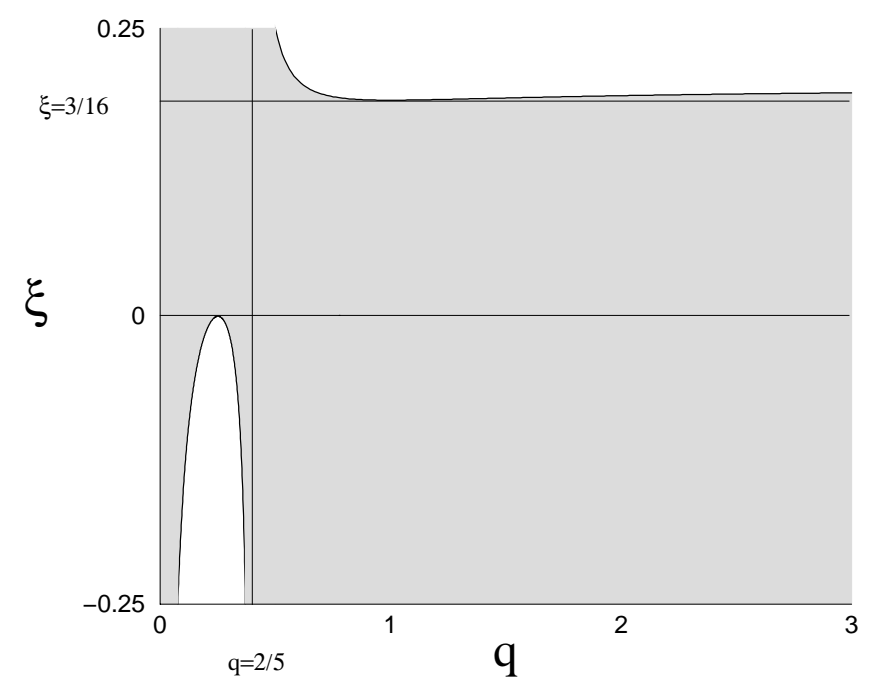

FIG. 1. The shaded region corresponds to the allowed values in the $(q-\xi)$ plane for a Dirichlet massless scalar field according to Eq. (86). Note that the range of $\xi$ comprised between the minimal and the conformal coupling is allowed for any value of $q$

In section $\mathrm{V}$ we concluded that the renormalized expression for the effective potential is

$$
V_{\theta}(\varphi)=\lim _{D \rightarrow 5}\left[V^{D}-\frac{1}{(D-5)} \frac{1}{\mathcal{A}} a_{5 / 2}^{D}\left(P_{\theta}\right)\right] .
$$

Consider first $V^{D}$, given in Eq. (55). Performing the momentum integrations, we obtain

$$
V^{D}=-\frac{1}{2(4 \pi)^{2}}\left(4 \pi \mu^{2}\right)^{\epsilon / 2} \frac{1}{z_{-}^{4-\epsilon}} \Gamma(-2+\epsilon / 2) \tilde{\zeta}(\epsilon-4)
$$

where we have defined [7]

$$
\tilde{\zeta}(s)=\sum_{n} \tilde{m}_{n}^{-s}=\frac{s}{2 \pi i} \int_{\mathcal{C}} t^{-1-s} \ln F(t) d t
$$

This regularized expression for the effective potential is finite when the real part of $\epsilon$ is sufficiently large. In the last equation we have used that $F(t)$ has only simple zeros which are along the real axis. The closed contour of integration 
$\mathcal{C}$ runs along the imaginary axis, from $t=+i \infty$ to $t=-i \infty$, skipping the origin through an infinitesimal path which crosses the positive real axis, and the contour is closed at infinity also through positive real infinity.

Now the problem reduces to the computation of $\tilde{\zeta}$, which can be done in the same way as in the case discussed in [7]. Skipping the detailed derivation, we simply give the final result:

$$
\tilde{\zeta}(-4+\epsilon)=-2 \beta_{4}\left(1+\eta^{-4+\epsilon}\right)-2 \epsilon(\eta \tau)^{-2}\left(\mathcal{I}_{K}+\mathcal{I}_{I} \tau^{4}+\tau^{4} \mathcal{V}(\tau)\right)+O\left(\epsilon^{2}\right)
$$

where

$$
\beta_{4}=\frac{1}{128}\left(13-56 \nu^{2}+16 \nu^{4}\right)
$$

Here we have introduced

$$
\tau \equiv \frac{z_{<}}{z_{>}}=\left\{\begin{array}{l}
1 / \eta, \text { for } \quad q<1 \\
\eta, \text { for } q>1
\end{array}\right.
$$

to express the result for both $q>1$ and $q<1$ cases simultaneously, where $z_{>}$and $z_{<}$are the largest and the smallest of $z_{+}$and $z_{-}$, respectively. Note that $\tau<1$.
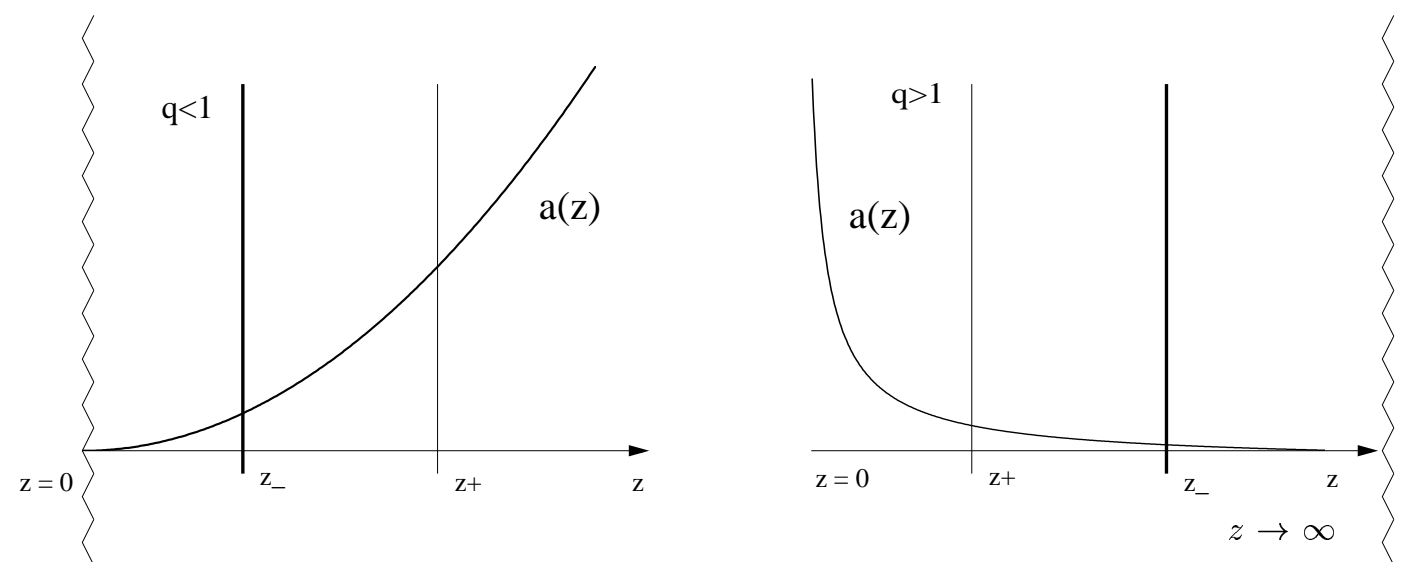

FIG. 2. For $q<1$, in conformal coordinate, the singularity sits at $z=0$, and the coordinate of the negative tension brane is smaller than the coordinate for the positive tension one, $\eta=z_{+} / z_{-}>1$. For $q>1$, the singularity is placed at $z \rightarrow \infty$ and $z_{-}>z_{+}$, so $\eta<1$.

The constant coefficients $\mathcal{I}_{K}(\nu), \mathcal{I}_{I}(\nu)$ are calculable in principle, although their precise value is perhaps not very interesting since as we shall see these coefficients can be reabsorbed by finite renormalization. Their definition is given in the Appendix. Finally, $\mathcal{V}(\tau)$ is defined by

$$
\mathcal{V}(\tau)=\int_{0}^{\infty} d \rho \rho^{3} \ln \left(1-\frac{I_{\nu}(\tau \rho)}{K_{\nu}(\tau \rho)} \frac{K_{\nu}(\rho)}{I_{\nu}(\rho)}\right)
$$

This provides the following expression for $V^{D}$

$$
\begin{aligned}
V^{D} & =\frac{1}{(4 \pi)^{2}}\left[\left\{\left(\frac{1}{\epsilon}+\frac{3}{4}-\frac{\gamma}{2}+\frac{1}{2} \ln \left(4 \pi \mu^{2} z_{0}^{2}\right)\right) \beta_{4}+\beta_{4}^{\prime}\right\}\left(\frac{1}{z_{-}^{4}}+\frac{1}{z_{+}^{4}}\right)\right. \\
& \left.+\beta_{4}\left(\frac{1}{z_{-}^{4}} \ln \left(\frac{z_{-}}{z_{0}}\right)+\frac{1}{z_{+}^{4}} \ln \left(\frac{z_{+}}{z_{0}}\right)\right)+\frac{\mathcal{I}_{K}}{z_{<}^{4}}+\frac{\mathcal{I}_{I}}{z_{>}^{4}}+\frac{\mathcal{V}(\tau)}{z_{>}^{4}}\right]+\mathcal{O}(\epsilon),
\end{aligned}
$$

where we have introduced the conventions $\beta_{4} \equiv \beta_{4}(D=5)$ and $\beta_{4}^{\prime} \equiv d \beta_{4}(D) /\left.d D\right|_{D=5}$, since $\beta_{4}$ depends on the dimension through (83).

The next step is to subtract the divergent contribution. The Seeley-De Witt coefficient $a_{5 / 2}^{D}\left(P_{\theta}\right)$ can be computed from (63) for a generic dimension $D$, so we can expand the second term in the r.h.s of Eq. (73) as 


$$
\frac{1}{(D-5)} \frac{1}{\mathcal{A}} a_{5 / 2}^{D}\left(P_{\theta}\right)=\frac{1}{(4 \pi)^{2}}\left[\frac{1}{\epsilon} \beta_{4}\left(\frac{1}{z_{-}^{4}}+\frac{1}{z_{+}^{4}}\right)-\beta \theta \beta_{4}\left(\frac{1}{z_{-}^{4}} \ln \left(\frac{z_{-}}{z_{0}}\right)+\frac{1}{z_{+}^{4}} \ln \left(\frac{z_{+}}{z_{0}}\right)\right)-\delta(\theta)\left(\frac{1}{z_{-}^{4}}+\frac{1}{z_{+}^{4}}\right)\right],
$$

where $\delta(\theta)$ is a constant whose precise value will be unimportant. ${ }^{7}$

The divergent parts of the two terms in Eq. (73) cancel, and the finite result for the effective potential per unit comoving volume is given by ${ }^{8}$

$$
\begin{gathered}
V_{\theta}=\frac{1}{(4 \pi)^{2}}\left[\frac{\mathcal{I}_{K}}{z_{<}^{4}}+\frac{\mathcal{I}_{I}}{z_{>}^{4}}+\frac{\mathcal{V}(\tau)}{z_{>}^{4}}\right]+\frac{\beta_{4}(\beta \theta+1)}{(4 \pi)^{2}}\left[\frac{1}{z_{+}^{4}} \ln \left(\frac{z_{+}}{z_{0}}\right)+\frac{1}{z_{-}^{4}} \ln \left(\frac{z_{-}}{z_{0}}\right)\right] \\
+\frac{\beta_{4}}{(4 \pi)^{2}}\left[\frac{1}{z_{+}^{4}}+\frac{1}{z_{-}^{4}}\right] \ln \left(\mu z_{0}\right) .
\end{gathered}
$$

Here, we have elliminated some terms through redefinition of $\mu$.

As in the case of conformal fields, we must also allow for finite renormalization of the couplings in front of the invariants which make up the coefficient $a_{5 / 2}$. These have the general dependence of the form $a_{ \pm}^{4} \mathcal{K}_{ \pm}^{4} \sim z_{ \pm}^{-4}$. With these additions, we finally obtain:

$$
V_{\theta}\left(z_{+}, z_{-}\right)=\frac{\beta_{4}(\beta \theta+1)}{(4 \pi)^{2}}\left[\frac{\ln \left(\mu_{1} z_{+}\right)}{z_{+}^{4}}+\frac{\ln \left(\mu_{2} z_{-}\right)}{z_{-}^{4}}\right]+\frac{1}{(4 \pi)^{2}} \int_{0}^{\infty} d x x^{3} \ln \left[1-\frac{I_{\nu}\left(x z_{<}\right)}{I_{\nu}\left(x z_{>}\right)} \frac{K_{\nu}\left(x z_{>}\right)}{K_{\nu}\left(x z_{<}\right)}\right]
$$

where $\mu_{1}$ and $\mu_{2}$ are renormalization constants, and $\beta$ and $\beta_{4}$ are given in equations (31) and (91). This is the main result of this Section. In the limit of small separation between the branes, $(1-\tau) \ll 1$, the integral $\mathcal{V}$ behaves like $(1-\tau)^{-4}$ (see Appendix C), and the logarithmic terms can be neglected. In this limit, the potential behaves like the one for the conformally coupled case, given in (33):

$$
V_{\theta}\left(z_{+}, z_{-}\right) \sim-\frac{A}{\left|z_{+}-z_{-}\right|^{4}}
$$

For $\tau \ll 1$, when the branes are well separated, the integral $\mathcal{V}$ behaves like $\tau^{2 \nu}$ and becomes negligible in the limit of small $\tau$ (except in the special case when $\nu$ is very close to 0 ). In this case, we have

$$
V_{\theta}\left(z_{+}, z_{-}\right) \sim \frac{\beta_{4}(\beta \theta+1)}{(4 \pi)^{2}}\left[\frac{\ln \left(\mu_{1} z_{+}\right)}{z_{+}^{4}}+\frac{\ln \left(\mu_{2} z_{-}\right)}{z_{-}^{4}}\right]+O\left[\left(\frac{z_{<}}{z_{>}}\right)^{2 \nu}\right] .
$$

Due to the presence of the logarithmic terms, it is in principle possible to adjust the parameters $\mu_{1}, \mu_{2}$ so that there are convenient extrema for the moduli $z_{+}$and $z_{-}$. We shall comment on this point in the concluding section.

\section{AN EXAMPLE}

Here we shall consider an example of a contribution to the moduli effective potential in the model of Lukas et al. $[1,22,23]$, which may be relevant for the Ekpyrotic universe [2]. As mentioned above, this corresponds to the case $q=1 / 6$. In principle, the fields in the action for this model do not have the canonical form, since in addition to the coupling to the metric they have unusual couplings to the dilaton $\phi$. Nevertheless, they can be studied along the lines of the previous Sections. For instance, the Heterotic M-theory model of $[1,22,23]$ contains a scalar $\Psi\left(x^{\alpha}\right)$ in the universal hypermultiplet whose vev is zero in the background solution, and whose action is given by

$$
S^{(\Psi)}=-\int \sqrt{g} d^{5} x \frac{1}{2} e^{-\phi}(\partial \Psi)^{2} .
$$

\footnotetext{
${ }^{7}$ For $\theta=1$, we find $\delta=(-\beta / 3072)\left\{432-1936 \beta-900 \beta^{2}+2257 \beta^{3}-1152(2+7(-1+\beta) \beta(1+3 \beta)) \xi+3072 \beta(-2+3 \beta)(-2+7 \beta) \xi^{2}\right\}$. ${ }^{8}$ We can check that this coincides with Eq. (34) in the conformal case, $\nu=1 / 2$ both for the Dirichlet or Neumann boundary conditions. The trace anomaly of conformally coupled fields in this conformally flat background is zero for this topology and for any kind of boundary conditions, and so $\beta_{4}=0$. Moreover, if $\nu=1 / 2$ then $K_{1 / 2}=\sqrt{\pi / 2 \rho} e^{-\rho}$ and $I_{1 / 2}=\sqrt{2 / \pi \rho}$ sinh $\rho$. This means that $\mathcal{I}_{K}, \mathcal{I}_{I}$ and $\mathcal{V}(\eta)$ can be computed analytically, easily recovering the result Eq. (34).
} 
This contains a kinetic term only, but it has a nonminimal form. However, changing to a new variable,

$$
\varphi=e^{-\phi / 2} \Psi
$$

we rewrite the action (100) as

$$
\begin{aligned}
S^{(\Psi)} & =-\int \sqrt{-g} d^{5} x \frac{1}{2}\left(\partial \varphi+\frac{1}{2} \varphi \partial \phi\right)^{2} \\
& =-\int \sqrt{-g} d^{5} x \frac{1}{2}\left(\partial \varphi^{2}-\frac{1}{2}\left(\square \phi-\frac{1}{2}(\partial \phi)^{2}\right) \varphi^{2}\right)+\text { boundary terms } \\
& =-\int a^{4} d^{4} x d y \frac{1}{2} \varphi\left(-\square-\frac{5}{12} \frac{1}{y^{2}}\right) \varphi,
\end{aligned}
$$

where the indices are contracted with the five dimensional $g_{a b}$ metric, and we have integrated by parts in the first equality. For the Dirichlet case, the boundary terms 'generated' are not relevant since still the field $\varphi$ vanishes there. The last equation shows that the potential term present in terms of $\varphi$ mimics a nonminimal coupling to the curvature $-\xi \mathcal{R} \varphi^{2}$. Since the Ricci scalar for this background is

$$
\mathcal{R}=\frac{7}{9} \frac{1}{y^{2}},
$$

we conclude that the equivalent effective nonminimal coupling of $\varphi$ is $\xi=15 / 28$. Note that this point lies in the allowed region of values in the $(q-\xi)$ plane defined by Eq. (86), which corresponds to an index of the Bessel functions $\nu=4 / 5$.

Hence, the contribution to the moduli effective potential induced by the field $\Psi$ is given by (97) with $\nu=4 / 5$. In this case we have $\beta=1 / 5$ and $\beta_{4}=-10179 / 80000$. There are, of course, many other contributions, corresponding to all bosonic and fermionic degrees of freedom. It turns out, however, that all bosonic contributions take a form similar to the one of the field $\Psi$ [24]. If supersymmetry is unbroken, then these contributions are cancelled by the contributions from the fermionic degrees of freedom. But if the degeneracy between bosons and fermions is broken a la Scherk-Schwartz, for instance, then we expect that the resulting effective potential will be qualitatively similar to the one given by (97). A detailed study of this model is left for future research.

\section{MODULI STABILIZATION}

In the limit of large interbrane separation, the potential (97) assumes a "Coleman-Weinberg" form for each one of the moduli,

$$
V\left(y_{+}, y_{-}\right) \approx \sum_{i= \pm} a^{4}\left(y_{i}\right)\left\{\alpha K^{4}\left(y_{i}\right) \ln \left[\frac{K\left(y_{i}\right)}{\mu_{i}}\right]+\delta \sigma_{i}\right\}
$$

Here, we have expressed the potential in terms of the "curvature scale"

$$
K(y)=\frac{q}{y},
$$

so that $K^{4}\left(y_{i}\right)$ behaves like a generic geometric operator of dimension 4 on the brane [such as the fourth power of the extrinsic curvature, or any of the operators in the integrand of Eq. (63)]. Working with $K_{i}$ instead of $z_{i}$ has the advantage of relating directly to physical quantities, and hence it is easier to control whether we are in the range where the effective theory should be trusted or not. In particular, we should not allow $K_{i}$ to be bigger than the cut-off scale of the theory. The constant $\alpha$ in (102) is given by

$$
\alpha=\frac{(1-\theta) q-1}{(4 \pi)^{2}}\left(1-q^{-1}\right)^{4} \sum \beta_{4}^{(\chi)},
$$

where we sum over the contributions from all bulk fields $\chi$. The numerical coefficients $\beta_{4}^{(\chi)}$ are given by Eq. (91). The value of $\theta$ depends on the choice of integration measure in the path integral which defines the effective potential (see Section IV). If we adopt the measure associated to the Einstein frame metric $g_{a b}$ which enters our original action functional (1), then we should take $\theta=1$. However, this is not the only possible choice, as we have repeatedly 
emphasized. The clasical action has a scaling symmetry which transforms both $g_{a b}$ and the background scalar field $\phi$. Using a conformal transformation which involves the scalar field, we may construct a new metric $g_{a b}^{(s)}$ which does not transform under scaling. If we adopt the measure which corresponds to this new metric, then we should take $\theta=1-1 / q$. With this particular choice of $\theta$ the coefficient $\alpha$ vanishes and the logarithmic terms in (102) disappear. Nevertheless, it is far from clear that this is indeed a preferred choice ${ }^{9}$ Here we take the attitude that the parameter $\theta$ is unknown, and that it should be fixed by a more fundamental theory of which (1) is just a low energy limit.

The renormalization constants $\mu_{i}$ in (102) can be estimated by looking at the "renormalized coefficient" of the geometric terms of dimension 4 on the brane $c_{i}(K)=\alpha \ln \left(K / \mu_{i}\right)$. In the absence of fine-tuning, the $c_{i}(K)$ are expected to be of order one near the cut-off scale $K \sim M$, where $M^{-3}$ is basically the five-dimensional Newton's constant. Hence, we expect

$$
\mu_{i} \sim M e^{-c_{i} / \alpha},
$$

where $c_{i}=c_{i}(M) \sim 1$. In (102), we have also allowed for finite renormalization of local operators on each one of the branes. These operators are collectively denoted by $\delta \sigma_{i}$. In order to ensure that the effective potential $V$ does not severely distort the background solution, this correction to the brane tension must be much smaller than the effective tension of the brane in the classical background solution. From the Darmois-Israel matching conditions, this effective tension is of order $M^{3} K_{i}$. Hence we require

$$
\delta \sigma_{i} \ll M^{3} K_{i} \ll M^{4} .
$$

In Section IV we considered contributions to the effective potential from massless bulk fields. These may have an arbitrary coupling to the curvature scalar of the standard form $\xi \mathcal{R} \chi^{2}$, or certain couplings to the background scalar field, such as the ones occurring in the Heterotic M-theory model considered in Section VI. However, if the model contains massive bulk fields, of mass $m$, then we expect terms proportional to $m^{2} K^{2}$ in the effective potential. Even without massive bulk fields, we may expect the presence of lower dimensional worldsheet operators of the form $M^{3} K$, $M^{2} K^{2}$ and $M K^{3}$, due to cubic, quadratic and linear divergences in the effective theory. Hence, we may expect that $\delta \sigma_{i}$ has an expansion of the form

$$
\delta \sigma_{i}\left(K_{i}\right) \sim \Lambda_{i}^{4}+\gamma_{1 i} M^{3} K_{i}+\gamma_{2 i} M^{2} K_{i}^{2}+\gamma_{3 i} M K_{i}^{3}+\gamma_{4 i} K_{i}^{4}+\mathcal{O}\left(K_{i}^{5}\right),
$$

where $K_{i} \ll M, \Lambda_{i} \ll M$ and $\gamma_{1 i} \ll 1$ in order to satisfy (105). For completeness, the above expansion includes the term proportional to $K_{i}^{4}$. It should be understood that this term is only present in the particular case $\theta=1-1 / q$ (corresponding to the scale invariant metric), since for other values of $\theta$ we assume that it is reabsorbed in a redefinition of $\mu_{i}$ [see Eq. (104)].

The local terms may in principle stabilize the moduli at convenient locations. Note that this effect is due to the warp factor and vanishes in flat space (where the coefficients $\beta_{4}$ vanish). The effect also vanishes accidentally in the RS case, because the curvature scale $K(y)$ is constant. The position of the minima are determined by $\partial_{y_{i}} V=0$. This leads to the conditions

$$
\delta \sigma_{i}=\frac{\alpha}{q} K_{i}^{4}\left[(1-q) \ln \left(\frac{K_{i}}{\mu_{i}}\right)+\frac{1}{4}\right]+K_{i} \frac{\delta \sigma_{i}^{\prime}}{4 q},
$$

where the prime on $\delta \sigma_{i}$ indicates derivative with respect to $K_{i}$. Also, we must require that the minima occur at an acceptable value of the effective cosmological constant. Using the condition (107), we can write the value of the potential at the minimum as

$$
V_{\min }=\frac{K_{+}^{4}}{4 q} \sum_{i= \pm}\left(\frac{K_{i}}{K_{+}}\right)^{4(1-q)}\left\{4 \alpha \ln \left(\frac{K_{i}}{\mu_{i}}\right)+\alpha+K_{i}^{-3} \delta \sigma_{i}^{\prime}\right\} \lesssim 10^{-122} m_{p}^{4}
$$

The latter condition will require one fine tuning amongst the parameters in (106).

\footnotetext{
${ }^{9}$ In particular, as mentioned in Section II, for certain values of the model parameters our action can be obtained by dimensional reduction of a $5+\mathrm{n}$ dimensional Einstein-Hilbert action with a cosmological term. Since in the higher dimensional theory only gravity is present, there is only one possible choice for the metric and the issue of the measure does not arise. Preliminary analysis of the case with $\mathrm{n}=1$ [24] indicates that the logarithmic terms do indeed arise in the limit when the radius of the sixth dimension is much smaller than the interbrane separation. For this case the relevant value of the parameter is $\theta=0$.
} 
An interesting question is whether the effective potential (102) can generate a large hierarchy and at the same time give sizable masses to the moduli. As discussed in Section II.B, the hierarchy is given by

$$
h^{2}=\frac{m^{2}}{m_{p}^{2}} \sim \frac{K_{+}}{M}\left(\frac{K_{+}}{K_{-}}\right)^{2 q-2},
$$

where $m \sim T e V$ is the mass of the particles which live on the negative tension brane, as perceived by the observers on the positive tension brane. Consistency with Newton's law at short distances requires $K_{+} \gtrsim(\mathrm{TeV})^{2} / \mathrm{m}_{p} \sim(\mathrm{mm})^{-1}$, and consistency of perturbative analysis requires $K_{-} \lesssim M$. With these constraints, the observed hierarchy $h \sim$ $\exp (-37)$ can only be accomodated for $q \gtrsim 5 / 4$. To proceed, we shall distinguish two different cases.

\section{A. Case $a$ :}

This is the generic case, where the coefficients $\gamma_{1 i}, \gamma_{2 i}$ and $\gamma_{3 i}$ in the expansion of $\delta \sigma_{i}(K)$ are not too suppressed. In this case, the logarithmic terms in the effective potential are in fact subdominant, and the minima of the effective potential are determined by $4 q \delta \sigma_{i} \approx K_{i} \delta \sigma_{i}^{\prime}$.

The present discussion applies also to the special case where $\theta=1-1 / q$ (corresponding to the measure associated with the scale invariant metric $\left.g_{a b}^{(s)}\right)$, so that no logarithmic terms are present in the effective potential. Note that terms of the form $\gamma_{4 i} K_{i}^{4}$ and $\gamma_{1 i} M^{3} K_{i}$ in the expansion of $\delta \sigma_{i}(K)$ [see (106)] cannot be avoided. The first is necessary in order to renormalize the effective potential, and the second is already present at the tree level, so it just corresponds to a shift in the existing parameters in the classical action.

Quite generically, this will lead to stabilization of the moduli near (or slightly below) the cut-off scale $K_{i}=\lambda_{i} M$, with $\lambda_{i} \sim 1$. Hence we have

$$
h^{2} \sim \exp \left[2(q-1) \ln \left(\lambda_{+} / \lambda_{-}\right)\right]
$$

Since the logarithm is of order one, an acceptable hierarchy can be generated provided that $q \gtrsim 10$. This is "close" to the RS limit $q \rightarrow \infty$. In this case, $m_{p} \sim M$. On the positive tension brane the parameter $\Lambda_{+}$has to be fine tuned so that the effective cosmological constant is 122 orders of magnitude smaller than the Planck scale. A straightforward calculation shows that the physical mass eigenvalues for the moduli $\varphi_{ \pm}$in the present case are given by

$$
m_{+}^{2} \sim q^{-2} m_{p}^{-2} K_{+}^{4} \lesssim m_{p}^{2}, \quad m_{-}^{2} \sim q^{-1} h^{2} m_{p}^{-2} K_{-}^{4} \lesssim h^{2} m_{p}^{2} .
$$

Thus, the lightest radion has a mass comparable to the $\mathrm{TeV}$ scale.

\section{B. Case b:}

This corresponds to the case where almost all of the operators in (106) are either extremely suppressed or completely absent, due perhaps to some symmetry. In particular, we shall concentrate on the possibility that

$$
\delta \sigma_{i}=\gamma_{1 i} M^{3} K_{i},
$$

since an operator of this form is already present in the classical action (1), and it is the only one in the expansion (106) which is allowed by the scaling symmetry. In this case, and assuming for simplicity that the negative tension brane is near the cut-off scale $K_{-} \sim M$, we can rewrite (108) as

$$
V_{\text {min }} \sim \frac{3 \alpha K_{+}^{4}}{(4 q-1)}\left\{\left(\ln \left(K_{+} / \mu_{+}\right)+\frac{1}{3}\right)+h^{8(q-1) /(2 q-1)}\left(\ln \left(K_{-} / \mu_{-}\right)+\frac{1}{3}\right)\right\}
$$

Here we are assuming that $\theta \neq 1-1 / q$ (so that $\alpha \neq 0$ ), since the alternative case was already discussed in the previous subsection. For $q>1$, the first term dominates and the condition of a nearly vanishing cosmological constant forces $K_{+} \approx \mu_{+} e^{-1 / 3}$. A fine-tuning of $\Lambda_{+}$will be necessary in order to satisfy the condition (107) for such value of $K_{+}$. The hierarchy is given by

$$
h^{2} \sim\left(\frac{\mu_{+}}{M}\right)^{2 q-1} \sim \exp \left[-(2 q-1) \alpha^{-1} c_{+}\right]
$$


where $\mu_{+}$is given by (104). Since the effective coupling $\alpha$ can be rather small, a large hierarchy may be obtained even for moderate $q \gtrsim 1$. A straightforward calculation shows that at the minima of the effective potential (102) $\partial_{\varphi_{+}}^{2} V=12 \alpha(1+2 q)^{-2} a_{+}^{4} K_{+}^{4} \varphi_{+}^{-2}$, and $\partial_{\varphi_{-}}^{2} V \sim \alpha q^{-1} a_{-}^{4} K_{-}^{4} \varphi_{-}^{-2}$. Hence, we find that the physical masses for the moduli fields $\varphi_{+}$and $\varphi_{-}$which appear in (15) are given by

$$
m_{+}^{2} \sim \alpha q^{-2} h^{12 /(2 q-1)} m_{p}^{2}, \quad m_{-}^{2} \sim \alpha q^{-1} h^{2+4 /(2 q-1)} m_{p}^{2} .
$$

Associated with the eigenvalue $m_{+}$there is a Brans-Dicke (BD) field ${ }^{10}$, with BD parameter $\omega_{B D}=-3 q /(1+2 q)$. Therefore, we must require $m_{+} \gtrsim(\mathrm{mm})^{-1}$, which in turn requires $q>2$. A stronger constraint on $q$ comes from the eigenvalue $m_{-}$, since the corresponding field is coupled to ordinary matter with $\mathrm{TeV}$ strength. The mass of this field cannot be too far below the $\mathrm{TeV}$, otherwise it would have been seen in accelerators. This requires $q$ to be rather large $q \gtrsim 10$.

\section{CONCLUSIONS}

We have studied a class of warped brane-world compactifications, with a power law warp factor of the form $a(y)=\left(y / y_{0}\right)^{q}$ and a dilaton with profile $\phi \propto \ln \left(y / y_{0}\right)$. Here $y$ is the proper distance in the extra dimension. In general, there are two different moduli $y_{ \pm}$corresponding to the location of the branes. (in the RS limit, $q \rightarrow \infty$, a combination of these moduli becomes pure gauge).

Classically, the moduli are massless, but they develop an effective potential at one loop. We have presented methods for calculating this effective potential, using both zeta function and in dimensional regularization. The procedure used in the case of zeta function regularization is a simpler version of the one we introduced in Ref. [7]. Our treatment of dimensional regularization formalizes and extends the approach adopted in Refs. [8-10]. An important point is that the divergent term to be subtracted from the dimensionally regularized effective potential is proportional to the the Seeley-de Witt coefficient $a_{5 / 2}$, given in (63). In the RS model, this coefficient behaves much like a renormalization of the brane tension, but it behaves very differently in the general case.

In general, the effective potential induced by massless bulk fields with arbitrary curvature coupling is given by (97). In the limit when the branes are very close to each other, it behaves like $V \propto a^{4}\left|y_{+}-y_{-}\right|^{-4}$, corresponding to the usual Casimir interaction in flat space. Perhaps more interesting is the moduli dependence due to local operators induced on the branes, which are the dominant terms in $V\left(y_{+}, y_{-}\right)$when the branes are widely separated. Such operators break a scaling symmetry of the classical action, which we discussed in Section II, but nevertheless are needed in order to cancel the divergences in the effective potential. If we denote by $K\left(y_{i}\right)=q / y_{i}$ the extrinsic curvature of the brane at the location $y=y_{i}(i= \pm)$, a renormalization of the brane tension parameters $\sigma_{ \pm}$in the classical action (1) induces terms proportional to $a\left(y_{i}\right)^{4} K_{i}$ in the effective potential. These terms scale like the rest of the classical action under the global transformation (19-20). On the other hand, the divergences in the effective potential, proportional to the coefficient $a_{5 / 2}(P)$, require world-sheet counterterms which are proportional to $a\left(y_{i}\right)^{4} K^{4}\left(y_{i}\right)$. These have the wrong scaling behaviour [they simply do not change under (19-20)] and hence they act as stabilizers for the moduli.

In addition, there are terms proportional to $a\left(y_{i}\right)^{4} K^{4}\left(y_{i}\right) \phi\left(y_{i}\right)$. The coefficient in front of the latter terms depends on the choice of the measure in the path integral. Different choices are possible, which are related amongst each other by dilaton dependent conformal transformations. Because of the conformal anomaly, different choices are inequivalent, but they are simply related by the addition of local world-sheet operators to the action. These are given by the r.h.s. of (82). Since the scaling symmetry of the action is anomalous, the presence of these local terms could perhaps have been guessed without the need of detailed calculation. Since $\phi$ behaves logarithmically, these terms have the form of Coleman-Weinberg type potentials for the moduli $y_{i}$, and they can also act as stabilizers for the moduli.

To conclude, we find that worldsheet operators induced on the brane at one loop easily stabilize the moduli in brane-world scenarios with warped compactifications, and give them sizable masses. If the warp factor is sufficiently steep, $q \gtrsim 10$, then this stabilization naturally generates a large hierarchy, as in the Randall-Sundrum model. In this case, the mass of the lightest modulus is somewhat below the $\mathrm{TeV}$ scale. This feature is in common with the Goldberger and Wise mechanism [25] for the stabilization of the radion in the RS model. For $q \lesssim 10$, the stabilization

\footnotetext{
${ }^{10}$ Here we are considering the situation where the mass of $\varphi_{-}$is much larger than the mass of $\varphi_{+}$, and where the visible matter is on the negative tension brane. In this case, since $y_{-}=$const., visible matter is universally coupled to the metric $g_{\mu \nu}$, and the BD parameter corresponding to $\varphi_{+}$can be read off from (15). Alternatively, it can be read off from (A4) after setting $y_{-}=$const.
} 
is also possible, but if we also demand that the hierarchy $h \sim 10^{-16}$ is generated geometrically, then the resulting masses for the moduli would be too low.

\section{ACKNOWLEDGEMENTS}

We are grateful to Klaus Kirsten and Alex Pomarol for useful discussions. J.G. thanks the Tufts Cosmology Institute for hospitality while part of this work was prepared. J.G. is partially supported by the Templeton Foundation under grant COS 253, by CICYT under grants AEN99-0766,AEN98-0431, by NATO under a collaborative research grant and by the Yamada Foundation. T.T. thanks the IFAE, where most of the present work was completed, for cordial hospitality. T.T. is partially supported by the Monbukagakusho Grant-in-Aid No. 1270154, and by the Yamada Foundation. O.P. is partially supported by CICYT, under grant AEN99-0766 and by CIRIT under grant 1998FI 00198 .

\section{APPENDIX A: ANOTHER FORM OF THE MODULI ACTION}

In this Appendix, we present alternative forms of the moduli action in terms of the metric induced on either brane. This highlights the fact that the interactions with matter on the branes are of Brans-Dicke type.

Changing variables in the background solution (2) according to $y=y_{-}+R \Theta$, with $0<\Theta<\pi$, we can recast the moduli as the radius of the orbifold $R$ and the 'would be' distance $y_{-}$from the negative tension brane to the singularity. Then the line element of our solution is

$$
d s^{2}=R^{2} d \Theta^{2}+\left(\frac{y_{-}+R \Theta}{y_{0}}\right)^{2 q} \eta_{\mu \nu} d x^{\mu} d x^{\nu}
$$

with $R$ and $y_{-}$arbitrary constants.

In order to take into account the gravitational degrees of freedom, we promote $R$ and $y_{\text {- }}$ (and the induced $4 \mathrm{~d}$ metric) to $4 \mathrm{~d}$ 'moduli' fields depending on $x^{\mu}$, so that the ansatz we shall consider takes the form

$$
\begin{aligned}
d s^{2} & =R\left(x^{\mu}\right)^{2} d \Theta^{2}+\left(1+r\left(x^{\mu}\right) \Theta\right)^{2 k} g_{\mu \nu}^{(-)} d x^{\mu} d x^{\nu}, \\
\phi & =\sqrt{6 q} \kappa_{5}^{1 / 2}\left[\ln \left(1+r\left(x^{\mu}\right) \Theta\right)+\ln \left(y_{-}\left(x^{\mu}\right) / y_{0}\right)\right]
\end{aligned}
$$

Here we have absorbed $y_{-}\left(x^{\mu}\right)$ into the $4 \mathrm{~d}$ metric $g_{\mu \nu}^{(-)}$, and defined $r \equiv R / y_{-}$. We insert this ansatz back to the action (1) and integrate over $\Theta$ to get the kinetic terms of the $4 \mathrm{~d}$ effective action. In terms of the metric on the negative tension brane we find

$$
\begin{aligned}
& S_{b}=\frac{-2}{\kappa_{5}} \int d^{4} x \sqrt{g_{(-)}} y_{-}\{\left(\frac{\lambda^{2 q+1}-1}{2 q+1}\right) \mathcal{R}^{(-)} \\
&\left.+3 q\left(\frac{\lambda^{2 q+1}-1}{2 q+1}\right) \frac{\left(\partial y_{-}\right)^{2}}{y_{-}^{2}}-3 q \lambda^{2 q+1} \frac{(\partial \lambda)^{2}}{\lambda^{2}}\right\},
\end{aligned}
$$

where indices are contracted with the $g^{(-)}$metric, $\mathcal{R}^{(-)}$is the $4 \mathrm{~d}$ curvature scalar related to it, and we defined

$$
\lambda \equiv\left(\frac{a(\pi)}{a(0)}\right)^{1 / q}=\frac{y_{+}}{y_{-}}=1+\pi r>1 .
$$

Note that $\lambda$ and $y_{-}$have diagonal kinetic terms, with $\lambda$ depending on the hierarchy of scales between the branes only.

The contribution to the kinetic part of the effective action from these moduli as seen from the positive tension brane is obtained from Eq. (A4) by writing $g_{\mu \nu}^{(-)}$as $\lambda^{-2 q} g_{\mu \nu}^{(+)}$(equivalently, we can change $\lambda$ by $1 / \lambda$ and $y_{-}$by $y_{+} \equiv \lambda y_{-}$). After partial integration and a global change of sign, we get

$$
\begin{aligned}
S_{b}=\frac{-2}{\kappa_{5}} \int d^{4} x \sqrt{g_{(+)}} y_{+}\{ & \left(\frac{1-\lambda^{-(2 q+1)}}{2 q+1}\right) \mathcal{R}^{(+)} \\
& \left.+3 q\left(\frac{1-\lambda^{-(2 q+1)}}{2 q+1}\right) \frac{\left(\partial y_{+}\right)^{2}}{y_{+}^{2}}+3 q \lambda^{-(2 q+1)} \frac{(\partial \lambda)^{2}}{\lambda^{2}}\right\},
\end{aligned}
$$


with indices contracted with $g^{(+)}$.

It is easy to see that both $\lambda$ and $y_{-}$or $y_{+}$can be put in the form of Brans-Dicke (BD) fields, with a generic action

$$
-\int d^{4} x \sqrt{g} \frac{1}{16 \pi}\left(\Phi_{B D} \mathcal{R}+\frac{\omega_{B D}\left(\Phi_{B D}\right)}{\Phi_{B D}}\left(\partial \Phi_{B D}\right)^{2}\right) .
$$

Ignoring the global $\lambda$-dependent factor, $y_{-}$and $y_{+}$are pure Brans-Dicke field up to a rescaling, with Brans-Dicke parameter $\omega_{B D}^{y_{ \pm}}=3 q$. In the case of $\lambda$, again ignoring the global $y_{-}$(or $y_{+}$) factor, the Brans-Dicke parameter are

$$
\omega_{B D}^{\lambda( \pm)}(\lambda)=\frac{3 q}{2 q+1}\left(\lambda^{ \pm(2 q+1)}-1\right) .
$$

From the positive tension brane, and for $q \sim 1$, the observational limit, $\omega_{B D}>3000$ [26], is fulfilled with $\lambda \sim 15$. This requires an interbrane proper distance $\pi R$ at least about 1 order of magnitude larger than the distance from the singularity at $y=0$ to the negative tension brane, at $y=y_{-}$. In the negative tension brane, we find that the BD parameter is negative but greater than $-3 / 2$ for any $\lambda$ or $q$, as happened in [27]. We reproduce the results of [28] in the limit of $q \rightarrow \infty$.

\section{APPENDIX B: DIMENSIONAL REGULARIZATION VERSUS ZETA FUNCTION REGULARIZATION}

\section{Zeta function regularization}

Let us rederive the main result of Section $\mathrm{V}$ using zeta function regularization. For simplicity, we shall restrict attention to the case $\theta=1$. In this case, we have explicitly calculated the integral (78) along the conformal path using both methods described in Section IV. This exercise leads to the result

$$
V=V_{0}-\frac{1}{(4 \pi)^{2}}\left[-\beta_{4}\left(\frac{1}{z_{-}^{4}} \ln \left(z_{-} / z_{0}\right)^{\beta}+\frac{1}{z_{+}^{4}} \ln \left(z_{+} / z_{0}\right)^{\beta}\right)+\hat{\alpha}\left(\frac{1}{z_{-}^{4}}+\frac{1}{z_{+}^{4}}\right)\right],
$$

where $\hat{\alpha}=(\beta / 3072)\left\{144+\beta\left(784-1692 \beta+335 \beta^{2}-192(3 \beta-14)(3 \beta-2) \xi\right)\right\}$. The zeta function associated with the operator $P_{0}$ is given by

$$
\zeta_{0}(s)=\mathcal{A} \int \frac{d^{4} k}{(2 \pi)^{4}} \sum_{i}\left(\frac{k^{2}+m_{n}^{2}}{\mu^{2}}\right)^{-s} .
$$

Performing the momentum integrals in (B2), we have

$$
\zeta_{0}(s)=\mathcal{A} \frac{\mu^{2 s} z_{-}^{2 s-4} \Gamma(s-2)}{(4 \pi)^{2} \Gamma(s)} \tilde{\zeta}(2 s-4),
$$

Substituting (B3) into (74), we have

$$
V_{0}\left(z_{+}, z_{-}\right)=-\frac{1}{2(4 \pi)^{2} z_{-}^{4}}\left[\left\{\ln \left(\mu z_{-}\right)+\frac{3}{4}\right\} \tilde{\zeta}(-4)+\tilde{\zeta}^{\prime}(-4)\right],
$$

and from (90) we obtain

$$
V_{0}\left(z_{+}, z_{-}\right)=\frac{1}{(4 \pi)^{2}}\left[\frac{\mathcal{I}_{K}}{z_{<}^{4}}+\frac{\mathcal{I}_{I}}{z_{>}^{4}}+\frac{\mathcal{V}(\tau)}{z_{>}^{4}}\right]+\frac{\beta_{4}}{(4 \pi)^{2}}\left[\frac{1}{z_{+}^{4}} \ln \left(\mu z_{+}\right)+\frac{1}{z_{-}^{4}} \ln \left(\mu z_{-}\right)\right] .
$$

Substituting in (B1) we recover Eq. (97) up to finite renormalization of $\mu$. 


\section{2. $V_{0}$ in dimensional regularization}

We shall now reproduce the result for $V_{0}$ by using dimensional regularization. Again, this is a redundant exercise: the calculation of an effective potential (be it $V$ or $V_{0}$ ) will give the same answer whether it is done in dimensional or in zeta function regularization. Nevertheless, it is interesting to do it explicitly since this calculation is closest in spirit to the standard flat space calculations in four dimensions.

Adding up the dimensionally regularized effective potential per comoving 4-volume due to all KK modes, we have

$$
V_{0}^{\mathrm{reg}}=\frac{1}{2} \mu^{\epsilon} \sum_{n} \int \frac{d^{4-\epsilon} k}{(2 \pi)^{4-\epsilon}} \log \left(\frac{k^{2}+m_{n}^{2}}{\mu^{2}}\right) .
$$

Performing the momentum integration for each mode, we obtain

$$
V_{0}^{\mathrm{reg}}=-\frac{1}{2(4 \pi)^{2}}\left(4 \pi \mu^{2}\right)^{\epsilon / 2} \frac{1}{z_{-}^{4-\epsilon}} \Gamma(-2+\epsilon / 2) \sum_{n} \tilde{m}_{n}^{4-\epsilon},
$$

where we used $\tilde{m}_{n}$ defined in (85). This regularized expression for the effective potential is finite when the real part of $\epsilon$ is sufficiently large. Performing analytical continuation in $\epsilon$, the summation over KK modes $\sum_{n} \tilde{m}_{n}^{4-\epsilon}$ can be identified with the zeta function $\tilde{\zeta}(-4+\epsilon)$. The pole part proportional to $1 / \epsilon$ is identified with

$$
V_{0}^{\text {div }}=-\frac{1}{\epsilon} \frac{1}{2(4 \pi)^{2} z_{-}^{4}} \tilde{\zeta}(-4) .
$$

Subtracting this divergent part, we get the renormalized expression for the effective potential as

$$
V_{0}=V^{\mathrm{reg}}-V^{\mathrm{div}}=-\lim _{\epsilon \rightarrow 0} \frac{1}{2(4 \pi)^{2}}\left[\frac{\left(4 \pi \mu^{2}\right)^{\epsilon / 2}}{z_{-}^{4-\epsilon}}\left(\frac{1}{\epsilon}+\frac{3}{4}-\frac{\gamma}{2}\right) \tilde{\zeta}(-4+\epsilon)-\frac{1}{\epsilon} \tilde{\zeta}(-4)\right] .
$$

Consequently, we find that the dimensional regularization method reproduces the previous result (B5).

Here, one remark is in order. In a usual 4-dimensional problem, the divergent part is given by the Seeley-De Witt coefficient $a_{2}$. In the present case, since the background is 4-dimensional flat space, $a_{2}$ consists of only one term proportional to $m_{n}^{4}$ for each KK mode. Subtraction of this counter term for each mode leads to the expression $\sum_{n} m_{n}^{4} \log m_{n}$, which is still divergent. This is not a surprising fact because the problem is essentially 5 -dimensional as it is indicated by the existence of the infinite tower of the KK modes. Usually, this point is bypassed in the literature by evaluating the divergent sum with the help of the generalized zeta function.

\section{Alternative regularization}

In section $\mathrm{V}$, we were interested in the class of conformally related operators $P_{\theta}$, and therefore it was important to do a dimensional extension of the spacetime such that all geometries labeled by $\theta$ would be conformally flat. In other words, we added dimensions "parallel" to the brane, whose "size" was also affected by the warp factor. It should be stressed that this was done for computational convenience, since $V^{D}$ is independent of the parameter $\theta$ only in this regularization. Putting aside computational considerations, nothing prevents us to extend the spacetime in any way we please, and the results should still be the same. To illustrate this point, let us consider an alternative dimensional extension of our 5-dimensional curved space $\mathcal{M}$ to a simple direct product space given by $\mathbb{R}^{-\epsilon} \times \mathcal{M}$. The dimensional regularization is done by an analytic continuation of the number of added dimensions $\epsilon$, while the manifold $\mathcal{M}$ is kept unchanged.

Since this is a direct product space, the eigenvalues of the $(5-\epsilon)$-dimensional d'Alembertian are given by a simple summation of those in each space, $\mathcal{M}$ and $\mathbb{R}^{-\epsilon}$,

$$
\lambda_{(5-\epsilon)}=k_{\omega}^{2}+\lambda
$$

Then, the dimensionally regularized effective potential per unit comoving volume is given by

$$
V^{\mathrm{reg}}=\frac{1}{2} \mu^{\epsilon} \sum_{\lambda} \int \frac{d^{-\epsilon} k_{\omega}}{(2 \pi)^{-\epsilon}} \log \left(\frac{k_{\omega}^{2}+\lambda}{\mu^{2}}\right)
$$


This quantity is evaluated by introducing the function

$$
\Upsilon(s)=\sum_{\lambda} \int \frac{d^{-\epsilon} k_{\omega}}{(2 \pi)^{-\epsilon}}\left(\frac{k_{\omega}^{2}+\lambda}{\mu^{2}}\right)^{-s}=(1 / 4 \pi)^{-\epsilon / 2} \frac{\Gamma(s+\epsilon / 2)}{\Gamma(s)} \zeta_{1}(s+\epsilon / 2),
$$

where $\zeta_{1}$ is the zeta function defined in (58) with $\theta=1$. Then, using (B11), we can rewrite (B10) as

$$
V^{\mathrm{reg}}=-\frac{1}{2 \mathcal{A}} \Upsilon^{\prime}(0)=-\frac{1}{2 \mathcal{A}}(1 / 4 \pi)^{-\epsilon / 2} \Gamma(\epsilon / 2) \zeta_{1}(\epsilon / 2) .
$$

The above expression contains the object $\zeta_{1}$, which we have encountered in zeta function regularization. However, it should be noted that now the regularization parameter is not $s$, the argument of the function $\Upsilon$, but the dimension of the product space $\epsilon$. Therefore, even after we take the limit $s \rightarrow 0, V^{\text {reg }}$ still diverges as $1 / \epsilon$.

The divergent piece in the dimensional regularization in the $D$ dimensional problem is the Seeley-De Witt coefficient $a_{D / 2}$. From (58) and (59), it is easy to relate this divergent piece with the value of $\zeta_{1}(0)$ as ${ }^{11}$

$$
V^{\operatorname{div}}=\frac{-a_{5 / 2}\left(P_{1}\right)}{\epsilon \mathcal{A}}=\frac{-1}{\epsilon \mathcal{A}} \zeta_{1}(0) .
$$

Subtracting this divergent piece from (B12), we obtain the renormalized value

$$
V=V^{\mathrm{reg}}-V^{\mathrm{div}}=-\frac{1}{2 \mathcal{A}}\left(\zeta_{1}^{\prime}(0)-\{\ln (1 / 4 \pi)+\gamma\} \zeta_{1}(0)\right),
$$

where $\gamma$ is Euler's gamma. This coincides with Eq. (74) for $\theta=1$ up to a redefinition of $\mu$ (note that with our conventions, $\zeta_{1}^{\prime}(0)$ depends on $\mu$.)

\section{APPENDIX C: DEFINITION OF THE CONSTANTS $\mathcal{I}_{I}$ AND $\mathcal{I}_{K}$}

The result $(90)$ involves the two constants $\mathcal{I}_{I}$ and $\mathcal{I}_{I}$. Although the actual value of this constants seems to be irrelevant, since they can be shifted by finite renormalization of worldsheet operators, we will nevertheless for the sake of completeness give their definition in terms of the parameters of the model.

We can express the asymptotic expansion of the combinations of modified Bessel functions used in subsection IV as

$$
\begin{aligned}
& I_{\nu}(\rho) \sim \frac{e^{\rho}}{\sqrt{2 \pi \rho}} \sum_{r=0}^{\infty} \frac{\delta_{r}^{(I)}}{\rho^{r}}+O\left(e^{-\rho}\right), \\
& K_{\nu}(\rho) \sim \sqrt{\frac{\pi}{\rho}} e^{-\rho} \sum_{r=0}^{\infty} \frac{\delta_{r}^{(K)}}{\rho^{r}} .
\end{aligned}
$$

Since both $\delta_{0}^{(I)}=\delta_{0}^{(K)}=1$, and the obsevration that $\beta_{4}^{(K)}=\beta_{4}^{(I)} \equiv \beta_{4}$, we can define coefficients $\beta_{r}$ for both $K_{\nu}$ and $I_{\nu}$ as

$$
\ln \left(\sum_{r=0}^{\infty} \frac{\delta_{r}}{\rho^{r}}\right)=\sum_{r=1}^{\infty} \frac{\beta_{r}}{\rho^{r}}
$$

Defining the functions

$$
\begin{aligned}
& R^{(K)}(\rho)=\sum_{r=1}^{3} \frac{\beta_{r}^{(K)}}{\rho^{r}}+\frac{\beta_{4}}{\rho^{4}} e^{-1 / \rho} \\
& R^{(I)}(\rho)=\sum_{r=1}^{3} \frac{\beta_{r}^{(I)}}{\rho^{r}}+\frac{\beta_{4}}{\rho^{4}} e^{-1 / \rho}
\end{aligned}
$$

\footnotetext{
${ }^{11}$ Strictly speaking $a_{5 / 2}^{D}$ has to be evaluated in the regularized $(5+\epsilon)$-dimensional space. However, since the added $\epsilon$ dimensions are trivial, it is identical to $a_{5 / 2}\left(P_{1}\right)$.
} 
the coefficients $\mathcal{I}_{I}$ and $\mathcal{I}_{K}$ are defined as

$$
\begin{aligned}
& \mathcal{I}_{I}=-\gamma \beta_{4}+\int_{0}^{\infty} d \rho \rho^{3}\left(\ln \left[\sqrt{2 \pi \rho} e^{-\rho} I_{\nu}(\rho)\right]-R^{I}(\rho)\right), \\
& \mathcal{I}_{K}=-\gamma \beta_{4}+\int_{0}^{\infty} d \rho \rho^{3}\left(\ln \left[\sqrt{2 \rho / \pi} e^{\rho} K_{\nu}(\rho)\right]-R^{K}(\rho)\right) .
\end{aligned}
$$

\section{APPENDIX D: ASYMPTOTIC FORM OF $\mathcal{V}(\tau)$}

The behaviour of $\mathcal{V}(\tau)$ defined in $(92)$ for $\tau \ll 1$ is given by

$$
\mathcal{V}(\tau)=-\frac{2}{\nu \Gamma(\nu)^{2}}\left(\frac{\tau}{2}\right)^{2 \nu} \int_{0}^{\infty} d t t^{2 \nu+3} \frac{K_{\nu}(t)}{I_{\nu}(t)}+\mathcal{O}\left(\tau^{4} \ln \tau\right)
$$

This corresponds to a large separation between branes. In this limit the integral is generically negligible compared with the logarithmic terms in (97).

The limit of small separation between branes corresponds to $1-\tau \ll 1$. In this limit, the integral can be approximated by taking the arguments of the Bessel functions to be large. Using the asymptotic expansion

$$
\frac{I_{\nu}(\tau \rho) K_{\nu}(\rho)}{K_{\nu}(\tau \rho) I_{\nu}(\rho)} \sim e^{-2(1-\tau) \rho},
$$

we have

$$
\begin{aligned}
\mathcal{V} & \approx \int_{0}^{\infty} d \rho \rho^{3} \ln \left(1-e^{-2(1-\tau) \rho}\right) \\
& =-\frac{1}{2^{6}(1-\tau)^{4}} \int_{0}^{\infty} d x \frac{x^{4}}{e^{x}-1}=-\frac{3 \zeta(5)}{8(1-\tau)^{4}}
\end{aligned}
$$

In the second equality, we performed integration by parts and a change of variable. Here, $\zeta$ is the usual Riemann's zeta function $\zeta(5)=1.03693 \cdots$. Using the relation

$$
\zeta^{\prime}(-4)=\frac{3}{4 \pi^{4}} \zeta(5),
$$

and substituting in (97), we find that in the limit of small brane separation, the effective potential reduces to the one we had found in the massless conformally coupled case, given in equation (33):

$$
V\left(z_{+}, z_{-}\right) \sim-\frac{A}{\left|z_{+}-z_{-}\right|^{4}} .
$$

Equation (D4) is a particular case of a more general formula

$$
\zeta^{\prime}(2 n)=\frac{(2 n) !}{2^{2 n+1} \pi^{2 n}} \zeta(2 n+1),
$$

valid for positive integer $n$. This can be derived from the perhaps better known relation [29]

$$
\zeta(1-z)=2^{1-z} \pi^{-z} \zeta(z) \Gamma(z) \cos (\pi z / 2),
$$

by setting $z=2 n+1$ after differentiation of both sides of the equation with respect to $z$.

[1] A. Lukas, B. A. Ovrut, K. S. Stelle and D. Waldram, "The universe as a domain wall," Phys. Rev. D59, 086001 (1999) [hep-th/9803235]. 
[2] J. Khoury, B. A. Ovrut, P. J. Steinhardt and N. Turok, "The ekpyrotic universe: Colliding branes and the origin of the hot big bang," hep-th/0103239.

[3] N. Arkani-Hamed, S. Dimopoulos, G. Dvali, "The Hierarchy Problem and New Dimensions at a Millimeter," Phys.Lett. B429, 263 (1998) [hep-ph/9803315]; "Phenomenology, Astrophysics and Cosmology of Theories with Submillimeter Dimensions and TeV Scale Quantum Gravity," Phys. Rev. D59, 086004 (1999) [hep-ph/9807344]; "New Dimensions at a Millimeter to a Fermi and Superstrings at a TeV," I. Antoniadis, N. Arkani-Hamed, S. Dimopoulos, G. Dvali, Phys.Lett. B436, 257 (1998) [hep-ph/9804398].

[4] L. Randall and R. Sundrum, "A Large Mass Hierarchy from a Small Extra Dimension," Phys. Rev. Lett.83, 3370 (1999)

[5] W.D. Goldberger and M.B. Wise "Bulk fields in the Randall Sundrum compactification scenario," Phys. Rev. D60, 107505 (1999) [hep-ph/9907218]; T. Gherghetta and A. Pomarol (CERN), "Bulk fields and Supersymmetry in a slice of AdS", Nucl. Phys. B586,141 (2000) [hep-ph/0003129].

[6] T. Appelquist and A. Chodos, "Quantum Effects in Kaluza-Klein Theories," Phys. Rev. Lett. 50, 141 (1983); "The Quantum Dynamics of Kaluza-Klein Theories," Phys. Rev. D28, 772 (1983).

[7] J. Garriga, O. Pujolas and T. Tanaka, "Radion effective potential in the brane-world," [hep-th/0004109];

[8] W. D. Goldberger and I. Z. Rothstein, "Quantum stabilization of compactified AdS(5)," Phys. Lett. B 491 (2000) 339 [hep-th/0007065].

[9] D. J. Toms, "Quantised bulk fields in the Randall-Sundrum compactification model," Phys. Lett. B 484 (2000) 149 hep-th/0005189

[10] A. Flachi and D. J. Toms, "Quantized bulk scalar fields in the Randall-Sundrum brane-model," hep-th/0103077

[11] See also S. Nojiri, S. Odintsov and S. Zerbini, Class. Quant. Grav. 17, 4855 (2000); I. Brevik, K. Milton,S. Nojiri and S. Odintsov, hep-th0010205.

[12] A.O. Barvinsky, "Braneworld effective action and the origin of inflation", hep-th/0107244.

[13] e.g. D. Youm, "Solitons in brane worlds," Nucl. Phys. B 576, 106 (2000) [hep-th/9911218];Nucl. Phys. B 596, 289 (2001) [hep-th/0007252].

[14] S.W. Hawking, Commun. Math. Phys. 56, 133 (1977); See also E. Elizalde, S. Odintsov, A. Romeo, A. Bytsenko and S. Zerbini "Zeta Regularization Techniques with Applications", World Scientific, Singapore, 1994; I Buchbinder, S.Odintsov and I. Shapiro, "Effective Action in Quantum Gravity", IOP Publishing, 1992.

[15] B.S. De Witt, "Quantum Field Theory in Curved Space-time" Phys. Rep. 19, 295 (1975).

[16] Kirsten, K., Spectral Functions in Mathematics and Physics, Chapman \& Hall/CRC, Boca Raton, FL, 2001; M. Bordag, E. Elizalde, K. Kirsten, "Heat kernel coefficients of the Laplace operator on the D-dimensional ball", J. Math. Phys. 37, (2) 895 (1996); K. Kirsten, "The a(5) heat kernel coefficient on a manifold with boundary," Class. Quant. Grav. 15, L5 (1998) [hep-th/9708081].

[17] I. Moss and J.S. Dowker, "The correct $B_{4}$ coefficient," Phys. Lett. B 229, 261 (1989).

[18] D.V. Vassilevich, "Vector fields on a disk with mixed boundary conditions," J. Math. Phys. 36, 3174 (1995).

[19] T.P. Branson, and P.B. Gilkey, "The asymptotics of the Laplacian on a manifold with boudary" Commun. Partial Diff. Eq. 15, 245 (1990).

[20] H.P. McKean Jr. and I.M. Singer, "Curvature and Eigenvalues of the Laplacian" J. Diff. Geom. 1, 43 (1967).

[21] J.S. Dowker and G. Kennedy, J. Phys. A 11, (1978); T. Branson and P.B. Gilkey, Trans. Am. Math. Soc. 344, (1994).

[22] P. Horava and E. Witten, "Heterotic and type I string dynamics from eleven dimensions," Nucl. Phys. B460, 506 (1996) [hep-th/9510209].

[23] P. Horava and E. Witten, "Eleven-Dimensional Supergravity on a Manifold with Boundary," Nucl. Phys. B475, 94 (1996) [hep-th/9603142].

[24] J. Garriga, O.Pujolas, T. Tanaka, in preparation.

[25] W. D. Goldberger and M. B. Wise, Phys. Rev. Lett. 83, 4922 (1999).

[26] C.M. Will, Theory and experiment in gravitational physics, revised edition, p123, Cambridge University Press, Cambridge, (1993); The confrontation between General Relativity and Experiment: A 1998 update, Lecture notes from the 1998 Slac Summer Institute on Particle Physics, gr-qc/9811036.

[27] J. Garriga and T. Tanaka, "Gravity in the Randall-Sundrum Brane World," Phys. Rev. Lett. 84, 2778 (1999) [hepth/9911055].

[28] T. Chiba, "Scalar-tensor gravity in two 3-brane system," Phys. Rev. D 62, 021502 (2000) [gr-qc/0001029].

[29] E. T. Whittaker, and G. N. Watson "A Course of Modern Analysis", Cambridge, UK: Univ. Pr. (1969) 608 p. 